\title{
Aves del Cañón del Chicamocha, Colombia: un llamado para su conservación
}

\author{
Birds of the Chicamocha Canyon, Colombia: a call for \\ conservation
}

Sergio Andrés Collazos-González ${ }^{(ㅁ}$, Juliana Zuluaga-Carrero ${ }^{(i)}$, José Oswaldo Cortés-Herrera ${ }^{(0)}$

\begin{abstract}
Resumen
Presentamos el listado de la avifauna actual del Cañón del Chicamocha ubicado entre los departamentos de Boyacá y Santander, Colombia. La región tiene una extensión de $3342 \mathrm{~km}^{2}$ y un gradiente altitudinal entre 300 y $2300 \mathrm{~m} \mathrm{s.} \mathrm{n.} \mathrm{m.} \mathrm{Los} \mathrm{datos} \mathrm{para} \mathrm{el} \mathrm{desarrollo} \mathrm{de} \mathrm{este} \mathrm{listado} \mathrm{fueron} \mathrm{recolectados} \mathrm{de} \mathrm{investigaciones} \mathrm{en} \mathrm{campo,}$ de la revisión de información secundaria y de registros en bases de datos. Se presenta un total de 291 especies de aves, entre las que se encuentran 239 residentes y 53 migratorias neárticas, de las cuales 8 se encuentran en alguna categoría de amenaza a nivel global y nacional, 7 son endémicas y 12 casi endémicas, destacándose el colibrí amazilia ventricastaño (Amazilia castaneiventris) y el cucarachero de Nicéforo (Thryophilus nicefori). Con esta información se aporta al desarrollo de iniciativas para la creación de áreas protegidas, fomento del aviturismo comunitario y proyectos de restauración ecológica.
\end{abstract}

Palabras clave. Avifauna. Bosque Seco Tropical. Especies focales. Listado taxonómico. Suramérica.

\begin{abstract}
We present a list of birds of the Chicamocha Canyon, located between the departments of Boyacá and Santander, Colombia. The region has an extension of $3342 \mathrm{~km}^{2}$ and an altitudinal gradient between 300 and $2300 \mathrm{~m}$ a. s. 1 . The dataset used in the development of this list was compiled from research carried out in the area, review of secondary sources, and review of databases. A total of 291 species of birds are presented, among which 239 are residents and 53 are Nearctic migrants; 8 of the species are in some threat category at a global and national level, 7 are endemic and 12 are almost endemic. The chestnut-bellied hummingbird (Amazilia castaneiventris) and Niceforo's wren (Thryophilus nicefori) are highlighted. This information contributes to the development of initiatives for the creation of protected areas, promotion of community birdwatching and ecological restoration projects.
\end{abstract}

Keywords. Birdlife. Focal species. South America. Taxonomic list. Tropical Dry Forest. 


\section{Introducción}

El enclave seco del Cañón del río Chicamocha tiene zonas áridas con una rica avifauna, lo que la ha convertido en un Área de Importancia para la Conservación de las Aves - AICA (Franco et al., 2009). Su orografía tiene un gradiente altitudinal entre 300 y $2300 \mathrm{~m} \mathrm{n}$. s. m, favoreciendo el establecimiento de variados hábitats para las aves. En la región se puede encontrar bosque seco tropical, bosque seco premontano, bosque de cañada, bosque de roble mixto, matorral xerofítico, humedales, helechales y afloramientos rocosos (Albesiano et al., 2003; Camargo-Ponce \& AgudeloÁlvarez, 2017). De igual manera, la marcada estacionalidad de la cordillera Oriental, ha permitido que algunas especies desarrollen adaptaciones particulares para sobrevivir a las condiciones extremas de sequía, por lo que se encuentra un alto número de endemismos en diferentes grupos taxonómicos en esta región (Hernández-Camacho et al., 1992; Pennington et al., 2006; Chaparro-Herrera et al., 2013).

Lamentablemente, solo un 20 \% del área se encuentra protegida bajo alguna figura de conservación de biodiversidad (Franco et al., 2009; Collazos-González \& Echeverry-Galvis, 2017), considerándose una región vulnerable a la presión humana (Etter et al., 2017; http:/ / datazone.birdlife.org). En el área aún se encuentran remanentes de bosque seco tropical (BST), un ecosistema fuertemente amenazado por la fragmentación y consecuente pérdida de hábitat, lo que en última instancia afecta a las especies con algún grado de vulnerabilidad (Sánchez-Azofeifa et al., 2005; Miles et al., 2006; Portillo-Quintero et al., 2010; Pizano et al., 2014). Esta condición ha llevado a que extensas áreas del cañón se transformen, amenazando los hábitats y las especies que lo habitan (Delgadillo-Méndez \& DelgadilloMéndez, 2013), y alterando, además, el suministro de agua para las poblaciones humanas de la región. Estos tensores y limitantes hacen prioritario el establecimiento de estrategias integrales que protejan sus valores ecológicos y culturales (Delgadillo-Méndez \& DelgadilloMéndez, 2013).

A pesar de la marcada estacionalidad en su precipitación y las fuertes presiones antrópicas que han sufrido sus ecosistemas, aún es posible observar en el cañón numerosas especies que merecen ser conservadas, por el aporte que pueden tener a la funcionalidad de los mismos (Albesiano \& Fernández, 2006; Valencia-Duarte et al., 2012; Etter et al., 2017). En el cañón se pueden encontrar remanentes de vegetación en los cuales hay especies de aves endémicas y amenazadas, entre las cuales se encuentran dos especies focales: el cucarachero de Nicéforo (Thryophilus nicefori) y el colibrí amazilia ventricastaño (Amazilia castaneiventris) (CortésHerrera et al., 2016; Collazos-González \& EcheverryGalvis, 2017). No obstante, a pesar de las condiciones únicas de este territorio, la información disponible acerca de la ecología de sus aves es escasa, de modo que es necesario continuar construyendo conocimiento en este tema y, así plantear herramientas para reducir la vulnerabilidad de las especies (Pizano et al., 2014, Collazos-González \& Echeverry-Galvis, 2017).

En el Cañón del Chicamocha se han llevado a cabo algunos estudios publicados e inéditos que aportan a la comprensión de su avifauna (Valderrama, 2005, Chaves-Portilla \& Cortés-Herrera, 2006; Vargas, 2007; Valderrama et al., 2008; Parra et al., 2010; Gómez \& Robinson, 2014; Collazos-González, 2015, CollazosGonzález, 2016; Collazos-González \& EcheverryGalvis, 2017; Zuluaga-Carrero, 2017; Rico-Guevara, 2018; Orozco-Romero, 2018). Estos estudios permitieron construir el presente listado de especies y reconocer las áreas en las cuales hay mayores necesidades de investigación, así como identificar otras áreas de importancia que podrían requerir acciones concretas de conservación y restauración. Finalmente, esperamos que esta revisión adicione de forma relevante conocimiento a un territorio que está nominado a ser Patrimonio de la Humanidad (UNESCO, 2012), aportando información notable sobre el estado actual de su avifauna.

\section{Materiales y métodos}

\section{Área de estudio.}

El Cañón del Chicamocha se encuentra ubicado en la vertiente occidental de la cordillera Oriental de los Andes colombianos, entre los departamentos de Boyacá y Santander (Figura 1). Se extiende de sur a norte y pertenece al sistema occidental de la cuenca hidrográfica del río Magdalena (Franco et al., 2009). Hace parte de la provincia biogeográfica de valle medio del río Chicamocha, incluida en el distrito del Cañón del Chicamocha de la provincia geográfica norandina, región árida aislada que estuvo antiguamente unida con 
las sabanas y bosques secos del valle de Magdalena y el Caribe (Hernández-Camacho et al., 1992). Formado hace 46 millones de años, el Cañón del Chicamocha es uno de los 58 centros de endemismo del país, y cubre alrededor de $3342 \mathrm{~km}^{2}$ en una longitud de $244 \mathrm{~km}$ (UNESCO, 2012; Etter et al., 2017). Es uno de los enclaves secos más extensos de Colombia y contiene la mayor cantidad de especies de flora y fauna endémicas en zonas xerofíticas y subxerofíticas (Díaz-Merlano \& Roberton, 2013).

En este enclave árido interandino predominan las pendientes fuertes y suelos pedregosos superficiales que presentan procesos avanzados de erosión (Guzmán, 2015; Camargo-Ponce \& Agudelo-Álvarez, 2017). El clima está fuertemente afectado por la topografía, en la cual hay valles con una profundidad aproximada de dos kilómetros hacia la localidad del Tabacal y laderas de hasta $71^{\circ}$ de inclinación en otras localidades (Díaz-Merlano, 2013; Guzmán, 2015). Según la clasificación de Caldas-Lang (1962) comprende los pisos climáticos cálido árido en zonas bajas, cálido semiárido en alturas medias y templado semihúmedo en sus partes altas (Camargo-Ponce \& Agudelo-Álvarez, 2017). En las partes bajas en el bosque muy seco se registran precipitaciones entre 100 y $500 \mathrm{~mm}$ y en bosque seco entre 1000 y $2000 \mathrm{~mm}$, con temperaturas que oscilan entre 24 y $32^{\circ} \mathrm{C}$; además, en las partes húmedas (>1500 $\mathrm{m} \mathrm{s.} \mathrm{n.} \mathrm{m.)} \mathrm{las} \mathrm{temperaturas} \mathrm{pueden} \mathrm{estar} \mathrm{entre} 20 \mathrm{y}$ $24{ }^{\circ} \mathrm{C}$ (Albesiano et al., 2003; Valencia et al., 2012). Su estacionalidad está marcada por la distribución de lluvias, siendo los meses de octubre y noviembre los más húmedos del año (Guzmán, 2015).

La vegetación caducifolia del cañón se caracteriza por poseer plantas con hojas pequeñas, con uno o dos estratos de follaje, por lo que la capacidad de retención de humedad es limitada (Albesiano \& Rangel, 2006; Camargo-Ponce \& Agudelo-Álvarez, 2017). Predominan coberturas vegetales de matorrales microfilocrasicaule, matorrales nanófilos y pastizales áridos (Albesiano et al., 2003), comprende cinco zonas de vida principales: bosque seco tropical (Bs-T), bosque muy seco tropical (Bms-T), monte espinoso premontano (me-PM), bosque seco premontano (Bs-PM) y bosque húmedo premontano (Bh-PM; Holdridge, 1987). El área de estudio comprende un rango altitudinal entre los 300 m s. n. m. del río Suárez y Chicamocha, hasta los 1904 m s. n. m. en las cotas más altas en la Mesa de
Xéridas en Los Santos, Santander y Boavita (Boquerón), Boyacá (Figura 1 y Figura 2).

Recolección de datos. El listado se elaboró con datos obtenidos en varias localidades del cañón. Se realizó una síntesis de las observaciones hechas en diferentes localidades en esta región, en los años 2004 y 2008 (Cortés-Herrera, 2004; Cortés-Herrera, 2006; Valderrama et al., 2008; Parra et al., 2010) y en tres quebradas en la Mesa de Xéridas, en el departamento de Santander, en el año 2014 (Camargo-Ponce \& Agudelo-Álvarez, 2017; Collazos-González \& Echeverry-Galvis, 2017; ZuluagaCarrero, 2017; Figura 1 y Tabla 1).

Adicionalmente esta revisión está basada en la investigación de Zuluaga-Carrero (2017) y Collazos-González \& Echeverry-Galvis (2017), quienes obtuvieron una representatividad de muestreo del $94 \%$ y $79 \%$ para el municipio de Los Santos. En estos estudios se hicieron observaciones directas en campo, acompañadas con registros auditivos y, como método complementario, en algunas localidades se utilizaron redes de niebla con 18900 metros/hora/red (Collazos-González \& Echeverry-Galvis, 2017).

Con el fin de complementar el listado, revisamos información secundaria (Gómez \& Robinson, 2014; Collazos-González, 2015; Collazos-González, 2016; RicoGuevara, 2018; Orozco-Romero, 2018), e informes técnicos de organizaciones no gubernamentales (Serrano et al., 2009; Ciri et al., 2011; Melo et al., 2012; Pardo \& Moreno-Arias, 2018).

Adicionalmente, revisamos las bases de datos del Instituto Alexander von Humboldt (IAvH, 2017), del Instituto de Ciencias Naturales de la Universidad Nacional de Colombia (ICN, 2019), del Global Biodiversity Information Facility GBIF (Montealegre, 2018) y el portal web Xenocanto Foundation (Xenocanto, 2019; Tabla 1). Los registros se validaron verificando su distribución espacial y altitudinal, soportando algunos registros con audios o fotografías, para así determinar con certeza la presencia de la especie en el área de estudio. El listado fue ordenado filogenéticamente siguiendo la clasificación sugerida por el Comité de Clasificación de Aves de Sur América (SACC, 2019) y posteriormente corroborado con las guías y listados de aves de Colombia (Hilty \& Brown 1986; Avendaño et al., 2017; Ayerbe-Quiñones, 2018) y conversaciones personales con observadores expertos del país. 


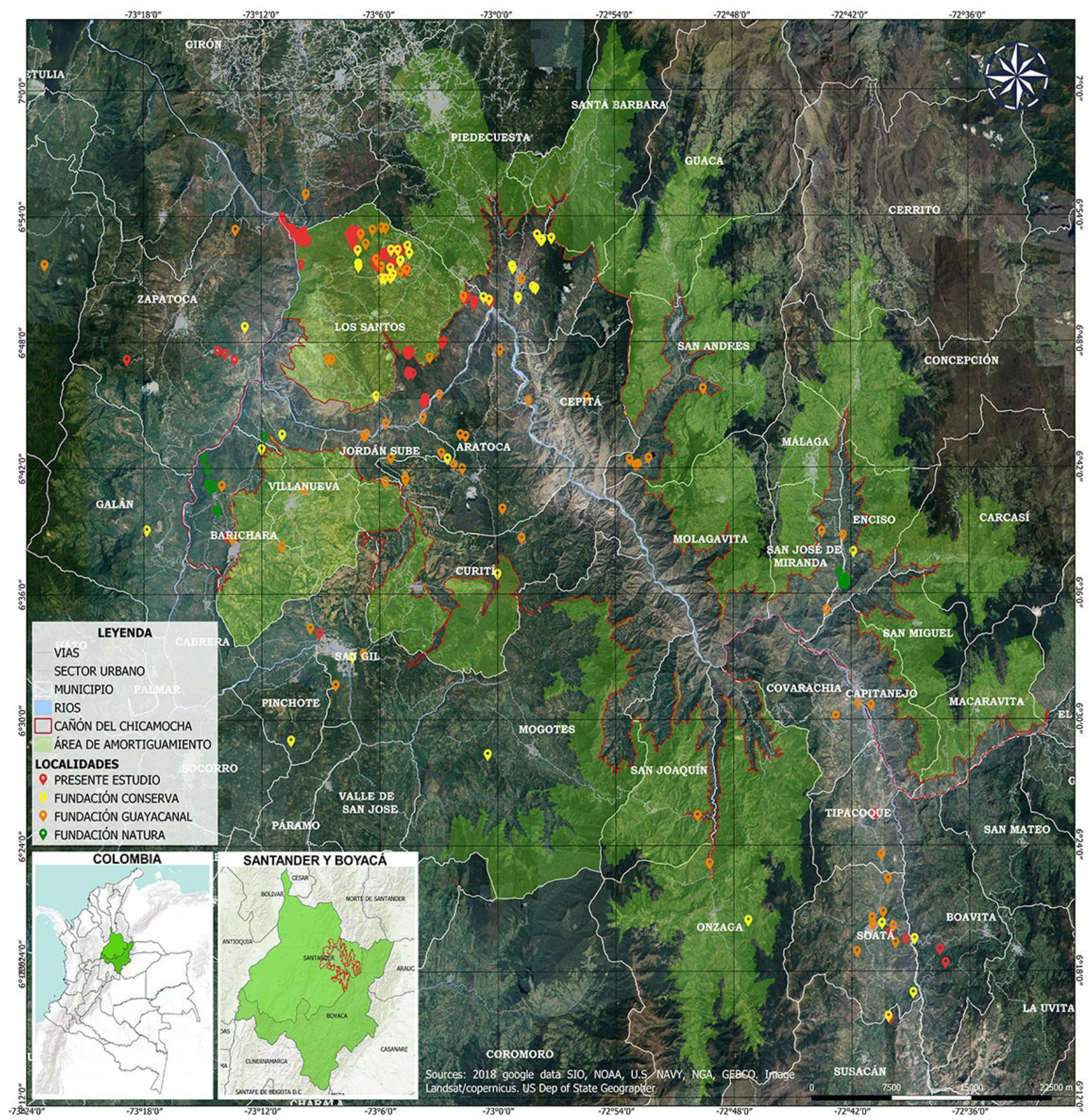

Figura 1. Localidades en las que se han realizado investigaciones sobre la avifauna del Cañón del Chicamocha, Colombia. 

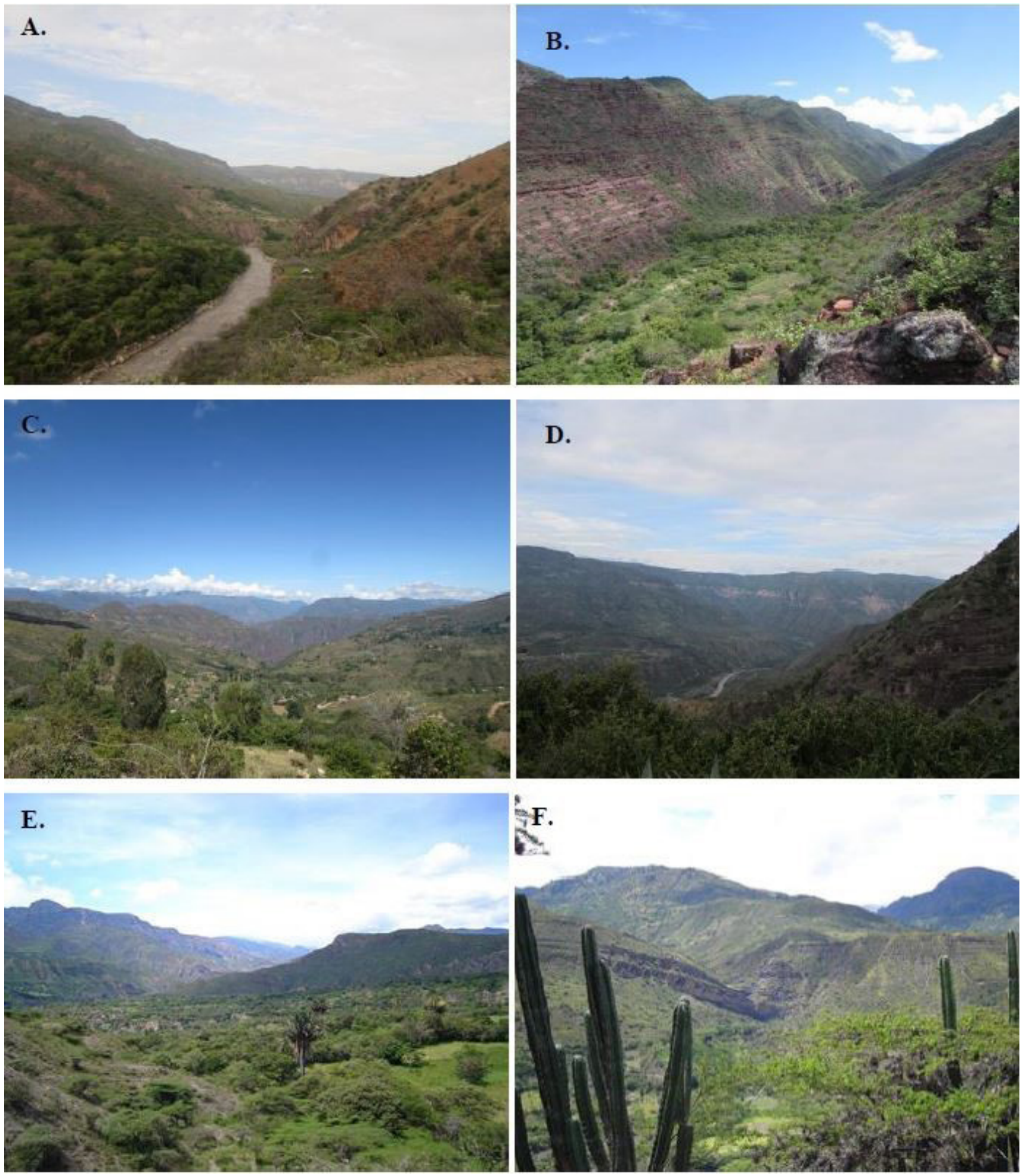

Figura 2. Paisajes de algunas localidades de estudio visitadas en el Cañón del Chicamocha, Colombia. Santander: A, JordánLos Santos; B, Los Fríos-Los Santos; C, La Purnia-Los Santos; D, La Mojarra-Los Santos. Boyacá: E y F, Soatá. Fotografías: Juliana Zuluaga-Carrero y Oswaldo Cortés-Herrera. 
Tabla 1. Localidades con registros de aves en el Cañón del Chicamocha, Colombia. Se incluye tanto la información de las bases de datos del presente estudio como otras localidades consultadas en: A, Instituto de Ciencias Naturales - ICN; B, Instituto Alexander von Humboldt - IAvH; C, Xeno-canto; D, Global Biodiversity Information Facility - GBIF.

\begin{tabular}{lllll}
\hline Municipio & Latitud & Longitud & Rango de elevación & Base de datos \\
\hline Aratoca & 6.4446 & -73.3190 & 900 & Presente estudio y A \\
\hline Barichara & 6.6403 & -73.2245 & $750-1300$ & Presente estudio, C y D \\
\hline Boavita & 6.1903 & -72.3731 & $1660-1904$ & Presente estudio, A y D \\
\hline Butaregua & 6.3955 & -73.1413 & $1300-1400$ & Presente estudio \\
\hline Curití & 6.3654 & -73.0000 & $1300-1500$ & Presente estudio \\
\hline Encino & 6.3627 & -72.4213 & $1350-1480$ & Presente estudio \\
\hline Galán & 6.3912 & -73.1750 & $900-950$ & Presente estudio \\
\hline Jordán & 6.4224 & -73.0231 & $400-450$ & Presente estudio \\
\hline Los Santos & 6.8846 & -73.1690 & $313-1649$ & Presente estudio, C y D \\
\hline Mogotes & 6.2819 & -73.0231 & $1700-1750$ & Presente estudio \\
\hline Onzaga & 6.2024 & -72.4716 & $1900-1953$ & Presente estudio \\
\hline Pescadero & 6.4959 & -73.0000 & 600 & Presente estudio y A \\
\hline Piedecuesta & 6.5919 & -73.0301 & $1000-1100$ & Presente estudio, A y B \\
\hline Pinchote & 6.2859 & -73.1031 & $1100-1131$ & Presente estudio \\
\hline San Gil & 6.3462 & -73.9437 & $1168-1200$ & Presente estudio, B y C \\
\hline Soatá & 6.1934 & -72.3915 & $1428-1639$ & Presente estudio, A, C y D \\
\hline Susacón & 6.1542 & -72.4044 & $1770-1800$ & Presente estudio \\
\hline Tipacoque & 6.2528 & -72.4046 & $1840-1900$ & Presente estudio \\
\hline Villanueva & 6.4333 & -73.1056 & $300-1450$ & Presente estudio \\
\hline Zapatoca & 6.8901 & -73.1769 & $317-1011$ & Prente estudio y C \\
\hline
\end{tabular}

Las especies con rango restringido las clasificamos de acuerdo a su estatus de endemismo (Chaparro-Herrera et al., 2013), como Endémica de Colombia (E) o Casi endémica de Colombia (CE). Los hábitos de migración y residencia fueron asignados según las características de orientación geográfica sugeridas por Naranjo et al. (2012) y Avendaño et al. (2017). Adicionalmente se añadieron al listado los nombres comunes en español según la plataforma web Avibase (2019).

\section{Resultados}

Se agruparon todos los registros de las especies correspondientes a 20 municipios (Tabla 1 y Figura 1). El municipio que contó con el mayor número de registros fue Los Santos, mientras que los municipios de Curití, Galán, Jordán, Mogotes, Onzaga, Pinchote, Tipacoque y Villanueva, cuentan con el menor número de observaciones. Se obtuvo un total de 291 especies para el Cañón del Chicamocha (Anexo 1), incluidas en 20 órdenes, 50 familias y 216 géneros. La avifauna está compuesta por 238 especies residentes, 53 especies migratorias, 51 de ellas migrantes latitudinales-transcontinentales boreales y dos australes, así como 20 especies que migran local y altitudinalmente (Naranjo et al., 2012).

Se registraron siete especies endémicas y doce casi endémicas, ocho de ellas en alguna categoría de amenaza a nivel nacional y mundial; dos Casi Amenazadas, cuatro Vulnerables, una En Peligro y una En Peligro Crítico (Renjifo et al., 2016). 
Las familias mejor representadas fueron Tyrannidae (49 especies, $16.2 \%)$, Thraupidae $(25,8.8 \%)$, Trochilidae $(25,8.8 \%)$ y Parulidae $(18,6.1 \%)$. Dentro de estas familias las especies con el mayor número de registros para el Cañón del Chicamocha, tanto en las investigaciones realizadas por los autores, como en las fuentes secundarias consultadas y bases de datos, fueron: Amazilia castaneiventris, Campylorhynchus griseus, Columbina talpacoti, Crotophaga ani, Dendroplex picus, Leptotila verreauxi, Momotus subrufescens, Myiarchus apicalis, Myiozetetes cayanensis, Setophaga pitiayumi, Stilpnia vitriolina, Thraupis episcopus, Thraupis palmarum, Tiaris bicolor, Todirostrum cinereum y Troglodytes aedon. Se encontró un menor número de registros para aves de hábitos nocturnos y especies acuáticas, lo que está relacionado con los limitados muestreos nocturnos, así como también con los pocos cuerpos de agua presentes en el área de estudio (Anexo 1).

\section{Discusión}

El listado de especies obtenido en esta revisión representa el $15 \%$ de las especies registradas para Colombia (Avendaño et al., 2017). El número de especies registradas hasta el momento para el Cañón del Chicamocha era de 104 (Parra et al., 2010), cifra que se incrementa considerablemente con el presente estudio alcanzando casi el triple de las especies. Así mismo, al comparar este listado con lo reportado para otros bosques secos en el país, encontramos que, se comparte un $70 \%$ de las especies de los valles interandinos en el departamento del Tolima, en donde se registran un total de 297 especies. De igual manera, en este listado se reporta el doble de las especies que han sido registradas para las franjas de bosque seco tropical entre los departamentos de Cundinamarca, Huila y Tolima, que documentan 145 especies (Gómez \& Robinson, 2014), un $37.7 \%$ más de las especies que han sido reportadas para el bosque seco tropical en el departamento del Cauca, con 212 especies (Ayerbe-Quiñones \& López-O, 2011), un $52 \%$ más de las especies reportadas recientemente para el bosque seco tropical de Norte de Santander, con 140 especies, y un 53\% más de las especies reportadas para el sur del Valle del Magdalena, con 127 especies (Avendaño et al., 2018; Espejo \& Morales, 2019). Considerando dichas cifras, se puede decir que el Cañón del Chicamocha es una de las regiones de bosque seco tropical más diversas en avifauna.
Al revisar los registros de los 20 municipios, se observaron especies que se encuentran en peligro de extinción en el país, como el colibrí amazilia ventricastaño (A. castaneiventris), especie categorizada En Peligro (EN) (Renjifo et al., 2016). Aunque esta especie es una de las que tuvieron un mayor número de registros, su distribución se restinge al área de estudio. Este colibrí fue redescubierto en el año 2003 en el municipio de Soatá, departamento de Boyacá (Cortés-Herrera, 2004). Para entonces, se desconocían sus rasgos de historia de vida, como preferencias alimenticias, requerimientos de hábitat, ecología y comportamiento. En algunos estudios, los registros de la especie permitían establecer un rango de distribución entre 850 y 2220 m s.n.m (Parra et al., 2010). Sin embargo, a partir de los registros obtenidos en este estudio y las observaciones que se han hecho, particularmente en la localidad de Los Santos, deducimos que esta especie se encuentra entre 120 y 2230 m s.n.m.

De igual manera encontramos registros para el cucarachero de Nicéforo (T. nicefori), especie categorizada en Peligro Crítico (CR) (Renjifo et al., 2016), contando con un número limitado de registros en el área de estudio. De esta especie endémica del cañón, hace unos años solo se conocía una población de pocos individuos en el municipio de San Gil, Santander (Valderrama et al., 2005; Parra et al., 2010), y según Collar et al. (1992) se estimaba que su rango altitudinal podría encontrarse entre 1050 y $1250 \mathrm{~m}$ s. n. m. En estudios más recientes, la especie ha sido registrada con mayor frecuencia en las zonas húmedas de los escarpes rocosos y los bosques de cañada de las laderas del cañón (Parra et al., 2016; Collazos-González \& Echeverry-Galvis, 2017), así como también hacia los bosques más húmedos de Zapatoca (Zuluaga-Carrero obs. pers). Esta información nos permite, por una parte confirmar la distribución de la especie en la localidad de San Gil, y por otra, indicar la extensión hacia las localidades de Los Santos, Galán, Zapatoca y Barichara, registros con los que planteamos una posible ampliación en su rango de distribución altitudinal a zonas más secas, que estaría desde los 680 hasta los $2100 \mathrm{~m} \mathrm{s.} \mathrm{n.} \mathrm{m.}$

Igualmente, en este extenso territorio hay otras especies endémicas para el país, entre ellas la guacharaca colombiana (Ortalis columbiana), el colibrí amazilia frentiazul (Amazilia cyanifrons), el atrapamoscas apical (Myiarchus apicalis) y la subespecie de cucarachero chupahuevos (Campylorhynchus griseus bicolor; Chaparro-Herrera et al., 2013; Figura 3). 
Otras subespecies de interés para la conservación son el pinzón alidorado (Arremon schlegeli canidorsum) y el turpial guajiro (Icterus icterus ridgwayi), que actualmente enfrentan un declive en sus poblaciones por pérdida y fragmentación de hábitat, y se encuentran en categoría Vulnerable (VU) (Renjifo et al., 2016). El pinzón alidorado es frecuentemente registrado en matorrales subxerofíticos en las laderas de los ríos Suárez, Chicamocha y Sogamoso; mientras que el turpial guajiro se observa constantemente en zonas secas de la Mesa de Xéridas en el municipio de los Santos y Piedecuesta, entre la cuenca media de los ríos Chicamocha y Sogamoso, alimentándose de especies de cactos como la pitahaya (Cereus hexagonus), el turmeperro (Opuntia stricta), el tuno (Opuntia schumannii) y el cardón (Stenocereus griseus) (Beltrán et al., 2016; Espejo et al., 2016; ZuluagaCarrero, 2017; S. Collazos obs. pers).

También, registramos especies casi endémicas, como periquito de anteojos (Forpus conspicillatus), zumbador ventriblanco (Chaetocercus heliodor), esmeralda de pico rojo (Chlorostilbon gibsoni), esmeralda colicorta (Chlorostilbon poortmani), amazilia verdiazul (Amazilia saucerrottei), ala de sable violeta (Campylopterus falcatus), batará crestibarrado (Thamnophilus multistriatus), tángara dorsirroja (Ramphocelus dimidiatus), tángara matorralera (Stilpnia vitriolina), gorrión montés cabecinegro (Arremon atricapillus), reinita gorgigrís (Myiothlypis cinereicollis) y jilguero andino (Spinus spinescens; Chaparro-Herrera et al., 2013; Avendaño et al., 2017).

Registramos 51 especies de migrantes transcontinentales del norte, destacándose la presencia del pibí boreal (Contopus cooperi) y la reinita cerúlea (Setophaga cerulea), migrantes boreales que se encuentran dentro de las categorías Casi Amenazada (NT) y Vulnerable (VU), respectivamente (BirdLife International, https:/ / www.birdlife.org/). De otro lado, solo obtuvimos registros de dos especies australes, el fiofío piquicorto (Elaenia parvirostris) y la tijereta sabanera (Tyrannus savana) (Naranjo et al., 2012).

Sobresale la presencia de poblaciones estables del atrapamoscas risquero (Hirundinea ferruginea) en zonas de altos escarpes que rodean el Cañón entre los 1300 y 1700 m s. n. m. (S. Collazos obs. pers; J. Zuluaga-Carrero obs. pers; Lopera-Salazar \& Chaparro-Herrera, 2017) y observaciones del águila paramuna (Geranoaetus albicaudatus) a $1495 \mathrm{~m} \mathrm{s.n.m}$. en los escarpes de la Mesa de Xéridas (Parra et al., 2010; S. Collazos obs. pers; F.
Ayerbe com. pers), registros muy por debajo de su rango de elevación para el país.

Encontramos un mayor número de localidades muestreadas en la literatura en el municipio de Los Santos, en el cual se registran el mayor número de especies, lo que probablemente sea resultado de un mayor esfuerzo de muestreo. En esta localidad se resaltan características como la heterogeneidad del paisaje, la diversidad de coberturas presentes a través de su gradiente altitudinal y la accesibilidad, que facilitan su estudio (Figura 2). Por otra parte, se debe continuar haciendo investigación en las localidades de Curití, Galán, Jordán, Mogotes, Onzaga, Peña Colorada, Pinchote, Tipacoque y Villanueva, en las cuales se encuentra un menor número de observaciones.

Las investigaciones realizadas por diferentes organizaciones estuvieron dirigidas al levantamiento de información de línea base de biodiversidad en varias zonas del Cañón del Chicamocha, muchas de ellas con el objeto de consolidar sitios de importancia para la conservación de la flora y fauna regional, teniendo como estrategia la creación de áreas protegidas (Parra et al., 2010). Se destacan los estudios para la declaración del Cañón del Chicamocha como área natural protegida, en jurisdicción de los municipios de Enciso, Capitanejo y San José de Miranda; la declaración de una área protegida de carácter público-regional y la formulación del plan de manejo del distrito de manejo integrado cañón del río Chicamocha en la subcuenca del río Umpalá, cuya información brinda oportunidades para la conservación de sus hábitats (Serrano et al., 2009; Ciri et al., 2011; Melo et al., 2012). No obstante, aún con estos trabajos las áreas protegidas dentro del Cañón siguen siendo mínimas para proteger especies focales y principalmente para el bosque seco tropical, el ecosistema más amenazado del país (Miles et al., 2006; Pizano et al., 2014; Etter et al., 2017).

Recientemente, se han llevado a cabo proyectos a distintas escalas, relacionados principalmente con la presión de transformación del área y su efecto en la riqueza de aves de la región (Camargo-Ponce \& AgudeloÂlvarez, 2017; Pardo \& Moreno-Arias, 2018). A partir de ellos, se han podido plantear como factores determinantes de la no persistencia de la avifauna, los procesos de erosión y escasez de agua, presiones antrópicas y la variabilidad ocasionada por el cambio climático (Collazos-González, 2016; Camargo-Ponce \& AgudeloÁlvarez, 2017; Orozco-Romero, 2018). Los proyectos mencionados han permitido plantear e implementar 

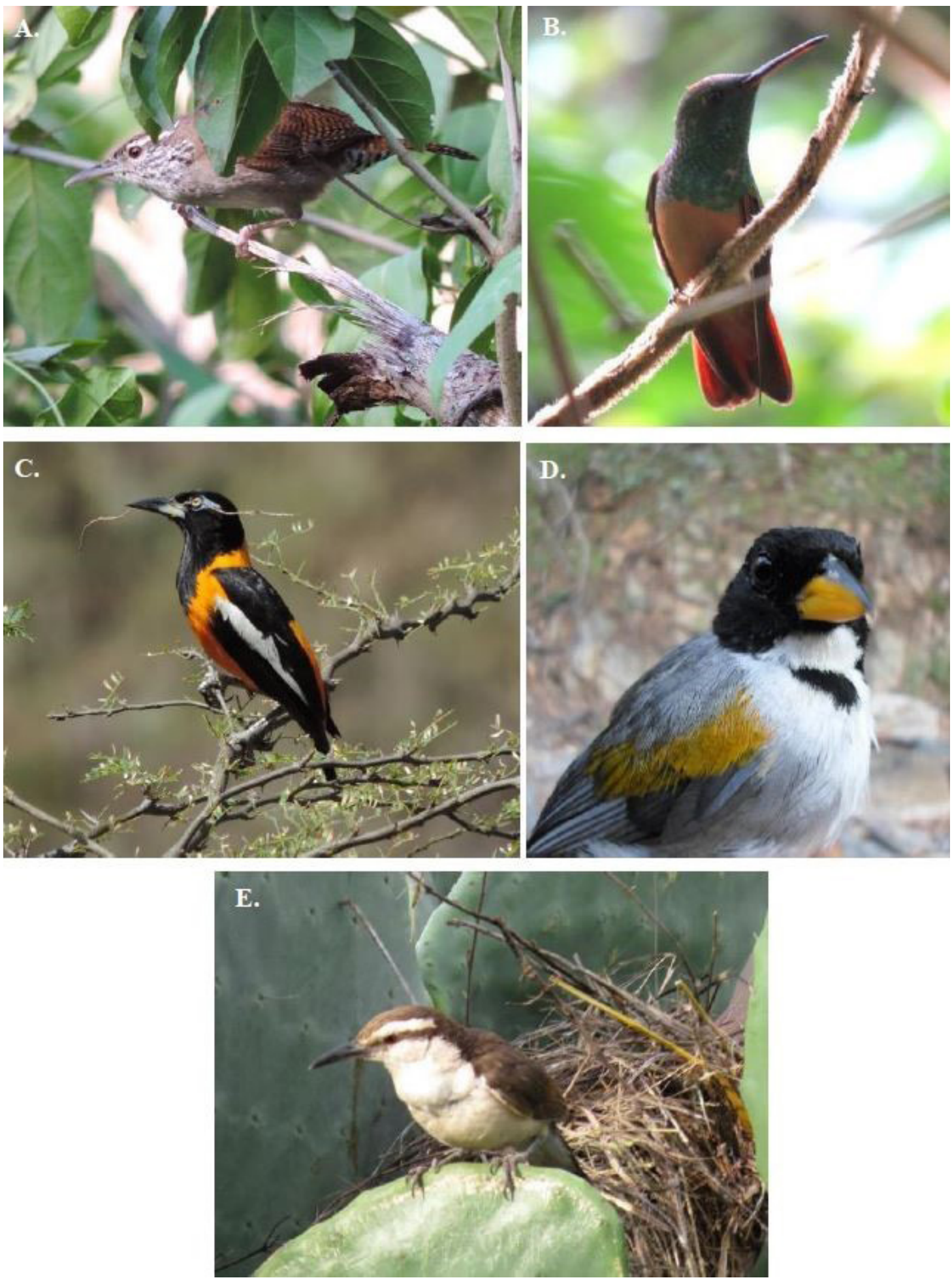

Figura 3. Especies focales de aves importantes para la conservación en el Cañón del Chicamocha, Colombia. A, cucarachero de Nicéforo (Thryophilus nicefori); B, amazilia ventricastaño (Amazilia castaneiventris); C, turpial guajiro (Icterus icterus); D, pinzón alidorado (Arremon schlegeli canidorsum); E, cucarachero chupahuevos (Campylorhynchus griseus bicolor). Fotografías: Sergio A. Collazos-González. 
iniciativas de manejo y uso, estrategias de restauración ecológica y monitoreos de biodiversidad (CollazosGonzález, 2015; Collazos-González, 2016; CamargoPonce \& Agudelo-Álvarez, 2017; Orozco-Romero, 2018). Es necesario ampliar la información, dirigiendo esfuerzos hacia el conocimiento ecológico e historia natural de las especies focales, con el objeto de disminuir sus amenazas (Piñeros-Quiceno, 2017). Asimismo, estas iniciativas deben incluirse en diferentes escenarios e instrumentos de planificación a partir de proyectos de educación ambiental y participación comunitaria (Hoyos et al., 2019).

Como lo definen Renjifo et al. (2016) y Piñeros-Quiceno (2017) es el momento de implementar iniciativas de gran alcance para llenar los vacíos en la representación de la biodiversidad, con el fin de garantizar la supervivencia de las especies amenazadas, incluyendo mayores esfuerzos en la creación de áreas protegidas en hábitats poco representados, como los bosques secos del valle del río Chicamocha, considerados AICA, y que cuenta con especies de aves prioritarias para la conservación (Franco et al., 2009). Proponemos que se inicien prioritariamente planes de manejo en las especies focales mencionadas, los cuales pueden ser desarrollados desde la academia, organizaciones locales, autoridades ambientales y ONGs. Igualmente, para que su divulgación y protección sea efectiva, se plantea el aviturismo comunitario rural como estrategia de construcción de oportunidades económicas y espacios para la conservación participativa de la avifauna del cañón (Maldonado et al., 2016). Dentro del marco de la iniciativa global Alianza para la Cero Extinción (ACE) y el AICA, consideradas como Áreas Clave para la Biodiversidad, se tendría un mayor alcance para dirigir iniciativas de conservación y manejo de hábitats, lo que aportaría al mantenimiento de los últimos remanentes de las poblaciones de especies evaluadas, como T. nicefori y A. castaneiventris (Cáceres et al., 2015; Piñeros-Quiceno, 2017).

El cañón del Chicamocha es un epicentro para comprender cómo la avifauna se mantiene en condiciones climáticas extremas y constantes presiones antrópicas. El cañón es potencialmente una de las áreas más diversas del país con remanentes de bosque seco, siendo prioritario establecer una figura regional o nacional de conservación que brinde protección a su biodiversidad. De igual forma, al albergar especies focales, ofrece mayores retos para comprender el estado de sus poblaciones, ya que con nuestros registros hemos evidenciado cambios en su distribución vertical. Es preciso tomar medidas para la planeación y el ordenamiento del territorio, incluyendo los actores que han configuradoel paisaje actual y que influyen e inciden en la distribución y permanencia de las aves de esta región.

\section{Agradecimientos}

Agradecemos a la Fundación Guayacanal, Fundación Conserva, Fundación Chimbilako y Ecopetrol por financiar gran parte del trabajo en campo de las investigaciones realizadas en el Cañón del Chicamocha. A los comentarios oportunos de los revisores anónimos y el equipo editorial. Reconocemos especialmente a todas las organizaciones públicas y privadas que con sus expediciones y estudios han permitido evidenciar y divulgar la biodiversidad de esta zona estratégica. Las capturas con redes de niebla se realizaron dentro del marco del permiso de estudio con fines de investigación científica Resolución 0284 de 2015 otorgado por la Autoridad Nacional de Licencias Ambientales (ANLA).

\section{Referencias}

Albesiano, S., Rangel-Ch, O. \& Cadena, A. (2003). La vegetación del cañón del río Chicamocha. SantanderColombia. Caldasia, 25(1), 73-99.

Albesiano, S. \& Rangel-Ch, O. (2006). Estructura de la vegetación del Cañón del río Chicamocha 500-1200 $\mathrm{m}$; Santander, Colombia: una herramienta para la conservación. Caldasia, 28(2), 307-325.

Avendaño, J., Bohórquez C., Rosselli, L., Arzuza-Buelbas, D., Estela, F., Cuervo, A., Stiles, G. \& Renjifo, L. (2017). Lista de chequeo de las aves de Colombia: Una síntesis del estado del conocimiento desde Hilty \& Brown (1986). Ornitología Colombiana, 16, eA01-2.

Avendaño, J.E., López-O, J.P. \& Laverde-R.O. (2018). Bird diversity of the Cúcuta valley (Colombia) and biogeographical affinities with dry forest avifaunas of northern South America. Wilson Journal of Ornithology, 130(1), 213-22.

Avibase (2019). The World Bird Database [Version online]. Available in: https:/ / avibase.bsc-eoc.org. Cited 10oct 2019. https:/ / doi.org/10.1676/16-016.1

Ayerbe-Quiñones, F. \& López-O, J.P. (2011). Adiciones a la avifauna del valle alto del río Patía, un área interandina en el suroccidente de Colombia. Boletín $S A O, 20,1-17$.

Ayerbe-Quiñones, F. (2018). Guía ilustrada de la avifauna colombiana. Serie Avifauna Colombiana. Bogotá D.C.: Wildlife Conservation Society. 458 pp. 
Beltrán, M. G., Parra, J.E. \& Delgadillo, A. (2016). Arremon schlegeli. En Renjifo, L., Amaya-Villarreal A., BurbanoGirón, J. \& Velásquez-Tibatá, J. (Eds). Libro rojo de aves de Colombia, Volumen II: Ecosistemas abiertos, secos, insulares, acuáticos continentales, marinos, tierras altas del Darién y Sierra Nevada de Santa Marta y bosques húmedos del centro, norte y oriente del país. Pp 380-381. Bogotá D.C.: Editorial Pontificia Universidad Javeriana e Instituto Alexander von Humboldt.

Cáceres, L. F., Moreno, C., Murillo, J. A. \& Briceño, E. R. (Eds.). (2015). Aves Amenazadas en el departamento de Santander. Estrategia regional para su conservación. San Gil, Colombia: Corporación Autónoma Regional de Santander (CAS).

Camargo-Ponce de León, G. \& Agudelo-Álvarez, L. (2017). Lectura de un paisaje estratificado: propuesta de restauración basada en el ordenamiento multiescala de las cañadas en la mesa de Xéridas, Santander, Colombia. Biota Colombiana, 18(2), 35-59. https:// doi.org/10.21068/c2017v18s01a02

Chaparro-Herrera, S., Echeverry-Galvis, M., CórdobaCórdoba, S. \& Sua-Becerra, A. (2013). Listado actualizado de las aves endémicas y casi-endémicas de Colombia. Biota Colombiana, 14(2), 113-150.

Chaves-Portilla, G. \& Cortés-Herrera, O. (2006). Nueva localidad para la quincha de Soatá (Amazilia castaneiventris) en el municipio de San Gil, Santander, Colombia. Boletín Sociedad Antioqueña de Ornitología, $16,1-6$.

Ciri, F., Saénz, F., Delgadillo, A., Ardila, F., Salamanca, R., Suescún, H. \& Díaz, E. (2011). Formulación del plan de manejo del distrito de manejo integrado cañón del río Chicamocha en la subcuenca del río Umpalá. Bogotá D.C.: Fundación Natura, CD-B Programa Conserva Colombia- FPAA, TNC, Fundación Neotropical.

Collar, N. J., Gonzaga, L. P., Krabbe, N., Madroño, A., Naranjo, L. G., Parker III, T. A. \& Wege, D. (1992). Threatened Birds of the Americas: The IUCN/ICBP Red Data Book. Cambridge, U.K.: International Council for Bird Preservation.

Collazos-González, S. A. (2015). Caracterización de la Avifauna presente en la Estación Biológica Guayacanal. (Informe técnico). Los Santos, Santander: Fundación Estación Biológica Guayacanal. 56 pp.

Collazos-González, S. A. (2016). Caracterización de la Avifauna presente en la Estación La Ceiba para la evaluación de procesos de restauración ecológica. (Informe técnico). Los Santos, Santander: Fundación Estación Biológica Guayacanal. 48 pp.

Collazos-González, S. \& Echeverry-Galvis, M. (2017). Comunidad de aves del bosque seco tropical en la mesa de Xéridas, Santander, Colombia. Ornitología Neotropical, 28, 223-235.

Cortés-Herrera, J. O., Hernández, A. J. \& Briceño, E. A. (2004). Redescubrimiento del colibrí Amazilia castaneiventris, una especie endémica y amenazada de Colombia. Ornitología Colombiana, 2, 47-49.

Cortés-Herrera, J. O. (2006). Aspectos ecológicos y de historia natural de Amazilia castaneiventris en Soatá (Boyacá). (Trabajo de grado). Bogotá D.C.: Universidad Distrital Francisco José de Caldas, Facultad de Ciencias y Educación, Licenciatura en Biología. 56 pp. Cortés-Herrera, J. O., Hernández-Jaramillo, A., ChávesPortilla, G., Villagrán-Chavarro, X. \& CastellanosMillán, A. (2007). Nuevos registros de poblaciones de aves amenazadas en Soatá (Boyacá), Colombia. Cotinga, 27, 74-77.

Cortés-Herrera, J. O., Chaves-Portilla, G. \& HernándezJaramillo, A. (2016). Amazilia castaneiventris. En Renjifo, L., Amaya-Villarreal, A., Burbano-Girón, J. \& Velásquez-Tibatá, J. (Eds). Libro rojo de aves de Colombia, Volumen II: Ecosistemas abiertos, secos, insulares, acuáticos continentales, marinos, tierras altas del Darién y Sierra Nevada de Santa Marta y bosques húmedos del centro, norte y oriente del país. Pp 223-226. Bogotá D.C.: Editorial Pontificia Universidad Javeriana e Instituto Alexander von Humboldt.

Delgadillo-Méndez, C. J. \& Delgadillo-Méndez, D. A. (2013). El cañón del Chicamocha: más que turismo, una reflexión sobre su biodiversidad. Revista Biodiversidad Colombia 1: 29-38.

Díaz-Merlano, J. \& Robertson, K. (2013). Cañones de Colombia. Cali, Colombia: Banco de Occidente Credencial. iM Editores. 196 pp.

Espejo, N. R., Zuluaga-Bonilla, J. E. \& Amaya-Villarreal, A. A. (2016). Icterus icterus. En Renjifo, L., Amaya-Villarreal, A., Burbano-Girón, J. \& Velásquez-Tibatá, J. (Eds). Libro rojo de aves de Colombia, Volumen II: Ecosistemas abiertos, secos, insulares, acuáticos continentales, marinos, tierras altas del Darién y Sierra Nevada de Santa Marta y bosques húmedos del centro, norte y oriente del país. Pp 401404. Bogotá D.C.: Editorial Pontificia Universidad Javeriana e Instituto Alexander von Humboldt.

Espejo, N. \& Morales, N. (2019). Variación de la diversidad taxonómica y funcional de la avifauna en un bosque seco tropical (bs-T) en diferentes estados de sucesión en el sur del Valle del Magdalena, Huila, Colombia. Caldasia, 41(1), 108-123. https:// doi.org/10.15446/caldasia.v41n1.71272

Etter, A., Andrade, A., Saavedra, K., Amaya, P. \& Arévalo, P. (2017). Estado de los Ecosistemas Colombianos: una aplicación de la metodología de la Lista Roja de 
Ecosistemas (Vers2.0). Informe Final. Bogotá D.C.: Pontificia Universidad Javeriana y Conservación Internacional Colombia. 138 pp.

Franco, A., Devenish, C., Barrero, M. \& Romero, M. (2009). Colombia. En Devenish, C., Díaz, D., Clay, R., Davidson, I. \& Yépez, I. (Eds.). Important Bird Areas Americas - Priority sites for biodiversity conservation. Pp 135-148. Quito, Ecuador: BirdLife International (BirdLife Conservation Series No. 16).

Gómez, J. \& Robinson, S. (2014). Aves del bosque seco tropical de Colombia: las comunidades del valle alto del río Magdalena. En Pizano, C. \& García, H. (Eds). El Bosque Seco Tropical en Colombia. Pp. 95-128. Bogotá D.C.: Instituto de Investigación de Recursos Biológicos Alexander von Humboldt.

Guzmán, G. (2015). The Chicamocha River Canyon. En Hermelín, M (Ed.). Landscapes and Landforms of Colombia. Pp. 73-83 Cambridge, UK: Springer International Publishing. https://doi.org/10.1007/978-3-319-11800-0_6

Hernández-Camacho, J. I., Hurtado, A., Ortiz, R. \& Walschburger, T. (1992). Centros de endemismo en Colombia. En Halffter, G. (Ed.). La diversidad biológica de Iberoamérica. Acta Zoológica Mexicana. Vol. Esp. 1992. Pp. 175-190. Xalapa, México: Programa Iberoamericano de Ciencia y Tecnología para el Desarrollo, Instituto de Ecología, A.C.

Hernández-Jaramillo, A., Cháves Portilla, G., VillagránChavarro, X., Castellanos-Millán, A. \& CortésHerrera, O. (2007). Estado y distribución actual de la Quincha de Soatá (Amazilia castaneiventris) especie endémica y en peligro crítico. Trabajo presentado en Programa y Libro de Memorias II congreso de Ornitología, Bogotá D.C., Colombia.

Hilty, S. \& Brown, W. (1986). A guide to the birds of Colombia. New Jersey, USA: Princeton University Press, Princeton. 1030 pp.

Holdridge, L. (1987). Ecología basada en zonas de vida. San José, Costa Rica: Instituto Interamericano de Ciencias Agrícolas. 216 pp.

Hoyos, M. A., Naranjo, L. G., Guerrero, J., Guevara, O., Suárez, C. F. \& Prüsmann, J. (2019). Conservación de la biodiversidad en un contexto de clima cambiante: experiencias de WWF Colombia en los últimos diez años. Biodiversidad en la Práctica, 4(1), 111-140.

Instituto Alexander von Humboldt -IAvH-. (2017). Colección de Aves de Colombia del Instituto. [Versión 6.0]. Disponible en: http://i2d.humboldt.org.co/.

ICN. Instituto Nacional de Ciencias Naturales. (2019). Colección en línea de aves. Disponible en: http:/ /www.biovirtual.unal.edu.co/en/collections.
López-Lanús, B. \& Cadena, C. D. (2002). Thryothorus nicefori. En Renjifo, L. M., Franco- Maya, A. M., Amaya-Espinel, J. D., Kattan, G. \& López-Lanús, B. (Eds.). Libro rojo de aves de Colombia. Pp 375-378. Bogotá D.C.: Serie libros rojos de especies amenazadas de Colombia. Instituto de Investigación de Recursos Biológicos Alexander von Humboldt y Ministerio del Medio Ambiente.

Lopera-Salazar, A. \& Chaparro-Herrera, S. (2017). Distribución del atrapamoscas roquero (Hirundinea ferruginea) en Colombia y nuevas localidades en la cordillera oriental. Boletín Sociedad Antioqueña de Ornitología, 26, 1 \& 2, 14-18.

Maldonado, J., Moreno-Sánchez, R., Espinoza, S., Bruner, A., Garzón, N. \& Myers, J. (2016). Resumen del estudio, La paz es mucho más que palomas: Beneficios económicos del acuerdo de paz en Colombia a partir del turismo de observación de aves. Bogotá D.C.: Conservation Strategy Fund. Serie Técnica N ${ }^{\circ}$ 46.

Melo, A., Ciri, F., Ramírez, A., Delgadillo, A., Díaz, C., Sáenz, F., Buitrago, C., Medina, C., Herrera, C., García, C., Parra, M., Alegría, F. \& Solano, C. (2012). Estudio para la declaración de un área protegida de carácter público - regional en el sector de bosque seco del cañón del Chicamocha en jurisdicción de los municipios de Enciso, Capitanejo y San José de Miranda, departamento de Santander. Bogotá D.C.: Fundación Natura - Programa Conserva Colombia - FPAA - TNC. 131 pp.

Meyer de Schauensee, R. (1946). A new species of wren from Colombia. Notulae Naturae, 182.

Miles, L., Newton, A., De Fries, R., Ravilious, C., May, I., Blyth, S., Kapos, V. \& Gordon, J. (2006). A global overview of the conservation status of tropical dry forests. Journal of Biogeography, 33, 491-505. https:// doi.org/10.1111/j.1365-2699.2005.01424.x

Montealegre, C (2018). Aves Bosque Seco Chicamocha. [Versión 2.0]. Fundación Natura Colombia. Occurrence dataset https://doi.org/10.15472/g0tc1y accessed via GBIF.org citado el 05 mar 2019.

Naranjo, L., Amaya-Espinel, J., Eusse-González, D. \& Cifuentes-Sarmiento, Y. (2012). Guía de las especies migratorias de la biodiversidad en Colombia. Volumen I: Aves. Bogotá D. C.: WWF-Colombia, Ministerio de Ambiente y Desarrollo Sostenible. 707 pp.

Organización de las Naciones Unidas para la Educación, la Ciencia y la Cultura - UNESCO. (2012). Cultural Landscape of the Lower Basin of the Chicamocha River [serial online] Disponible en: https://whc.unesco.org/en/tentativelists/5757. 
Orozco-Romero, D. (2018). ¿Las perchas artificiales simples para aves aumentan la lluvia de semillas en un pastizal del Bosque Seco Tropical en la Mesa de los Santos, Santander, Colombia? (Trabajo de grado). Bogotá D.C.: Pontificia Universidad Javeriana, Facultad de Estudios Ambientales y Rurales. 48 pp.

Pardo, M. E. \& Moreno-Arias, R. (Eds). (2018). El enclave seco del cañón del Chicamocha: biodiversidad y territorio. Bogotá D.C.: Fundación Natura.192 pp.

Parra, J. E., Beltrán, L. M., Delgadillo, A. \& Valderrama, S. (2010). Project Chicamocha II: Saving threatened dry forest biodiversity. (Final report). Bogotá D. C.: Conservation Leadership Programme. Disponible en: http:/ / www.conservationleadershipprogramme.org/

Parra, J. E., Valderrama, S., Delgadillo, A. \&. Beltrán, L. M. (2016). Thryophilus nicefori. En Renjifo, L., AmayaVillarreal, A., Burbano-Girón, J. \& VelásquezTibatá, J. (Eds). Libro rojo de aves de Colombia, Volumen II: Ecosistemas abiertos, secos, insulares, acuáticos continentales, marinos, tierras altas del Darién y Sierra Nevada de Santa Marta y bosques húmedos del centro, norte y oriente del país. Pp 358361. Bogotá D.C.: Editorial Pontificia Universidad Javeriana e Instituto Alexander von Humboldt.

Pennington, T., Gwilyn, P. \& Ratter, J. (2006). An overview of the plant diversity, biogeography and conservation of neotropical savannas and seasonally dry forest. En Pennington, T., Gwilyn, P. \& Ratter, J. (Eds) Neotropical Savannas and Seasonally Dry Forests. Plant Diversity, Biogeography and Conservation. Pp. 1-29. Boca Raton, Florida, USA: The Systematics Association. Special Volume Series 69. https://doi.org/10.1201/9781420004496-1

Piñeros-Quiceno, A. M. (2017). Incidencia de las listas rojas en la gestión para la conservación de las especies amenazadas a escalas global y nacional (Colombia). (Trabajo de grado) Bogotá D.C.: Pontificia Universidad Javeriana, Facultad de Estudios Ambientales y Rurales. $117 \mathrm{pp}$.

Pizano, C., Cabrera, M. \& García, H. (2014). Bosque seco tropical en Colombia; generalidades y contexto. En Pizano, C. \& García, H. (Eds.). El Bosque Seco Tropical en Colombia. Pp. 36-47. Bogotá: Instituto de Investigación de Recursos Biológicos Alexander von Humboldt (IAvH).

Portillo-Quintero, C. \& Sánchez-Azofeifa, G. (2010). Extent and conservation of tropical dry forests in the Americas. Biological Conservation, 143, 144-155. https:// doi.org/10.1016/j.biocon.2009.09.020

Renjifo, L.M., Amaya-Villarreal, A., Burbano-Girón, J. \& Velásquez-Tibatá, J. (2016). Libro Rojo de Aves de Colombia. Volumen II. Ecosistemas Abiertos, Secos,
Insulares, Acuáticos Continentales, Marinos, Tierras Altas del Darién y Sierra Nevada de Santa Marta y Bosques Húmedos del Centro, Norte y Oriente del País. Bogotá D.C.: Editorial Pontificia Universidad Javeriana e Instituto Alexander von Humboldt. 564 pp.

Rico-Guevara, A. (2018). Diversidad de aves en bosques secos del cañón de los ríos Chicamocha y Suárez (Santander, Colombia). En Pardo, M. E. \& MorenoArias, R. (Eds). El enclave seco del cañón de Chicamocha: biodiversidad y territorio. Pp. 16-22. Bogotá D.C.: Fundación Natura. 192 pp.

SACC. (2019). South American Classification Committee. [Versión online]. Disponible en: http://www.museum.lsu.edu.

Sánchez-Azofeifa, G., Quesada, M., Rodríguez, J., Nassar, J., Stoner K., Castillo A., Garvin T., Zent, E., CalcoAlvarado J., Kalacska M., Fajardo L., Gamon, J. \& Cuevas-Reyes, P. (2005). Research Priorities for Neotropical Dry forests. Biotropica, 37(4), 477-485. https:// doi.org/10.1046/j.0950-091x.2001.00153.x-i1 Serrano, M., Pacheco, B., Gualdrón, J., Nieto, O., Sánchez, I., Acosta, A., Beltrán, C. \& Gómez, F. (2009). Estudio básico para la declaratoria de un área natural protegida en el cañón del Chicamocha, Jurisdicción CDMB, Bucaramanga, Colombia. Bucaramanga, Colombia: Fundación para la conservación del patrimonio natural Biocolombia, Corporación autónoma regional para la defensa de la meseta de Bucaramanga CDMB. 283 pp.

Valderrama, S. V. (2005). Contribución al conocimiento de la historia natural, ecología y hábitat del cucarachero de Nicéforo en un enclave seco del municipio de San Gil (Santander). (Tesis de grado). Bogotá D.C.: Universidad de Los Andes, Departamento de Biología. 48 pp.

Valderrama, S. V., Parra, J. E., Dávila, N. \& Mennill, D. J. (2008). Vocal behaviour of the critically endangered Niceforo's wren (Thryothorus nicefori). The Auk, 125, 395-401. https://doi.org/10.1525/auk.2008.06249

Valderrama, S. V. \& Páez, C. A. (2009). Population status and ecological requirements of Niceforo's Wren in the Chicamocha Canyon, Colombia. (Final report). Bogotá D.C.: Fundación Proaves, The Royal Society for the Protection of Birds. 78 pp.

Valencia-Duarte, L., Trujillo, N. \& Vargas, O. (2012). Dinámica de la vegetación en un enclave semiárido del río Chicamocha, Colombia. Biota Colombiana, 13(2), 40-65.

Vargas-Moreno, K. (2007). Evaluación del estado taxonómico del cucarachero de nicéforo Thryothorus nicefori (Aves: Troglodytidae) mediante métodos morfológicos y genéticos. (Tesis de grado). Bogotá D.C.: Universidad de los Andes. 46 pp. 
Xeno-canto. (2019). Portal web Xenocanto Foundation [Versión Online]. Disponible en: https://www.xeno-canto.org.

Zuluaga-Carrero, J. (2017). Diversidad composicional y funcional de aves en diferentes tipos de vegetación durante el periodo de lluvias en el enclave seco del Cañón del río Chicamocha - Santander. (Trabajo de grado). Bogotá D.C.: Pontificia Universidad Javeriana, Facultad de Estudios Ambientales y Rurales. 88 pp. 
Anexo 1. Listado taxonómico de las aves del Cañón del Chicamocha, Colombia.

\begin{tabular}{|c|c|c|c|c|c|}
\hline Taxón & $\begin{array}{l}\text { Nombre en } \\
\text { Español }\end{array}$ & $\begin{array}{l}\text { Categorías de resi- } \\
\text { dencia, endemici- } \\
\text { dad y amenaza }\end{array}$ & $\begin{array}{c}\text { Obs. } \\
\text { Autores }\end{array}$ & Fuente & $\begin{array}{c}\text { Bases de } \\
\text { datos }\end{array}$ \\
\hline \multicolumn{6}{|l|}{ Tinamidae } \\
\hline Nothocercus bonapartei (Bonaparte, 1854) & Tinamú serrano & & O.C & & \\
\hline Crypturellus soui (Hermann, 1783) & Tinamú chico & & O.C & & $\mathrm{C}$ \\
\hline \multicolumn{6}{|l|}{ Anatidae } \\
\hline Dendrocygna viduata (Linnaeus, 1766) & Sirirí cariblanco & & O.C & & \\
\hline Anas discors (Linnaeus, 1766) & Cerceta aliazul & Lat, Trans, Irp, Mb & S.C-O.C-J.Z & 4,17 & \\
\hline \multicolumn{6}{|l|}{ Cracidae } \\
\hline Chamaepetes goudotii (Lesson, 1828) & Pava falcialar & & O.C & & \\
\hline Penelope argyrotis Bonaparte, 1856 & Pava camata & & S.C-O.C-J.Z & $2,4,5,23$ & $\mathrm{C}$ \\
\hline Ortalis columbiana Hellmayr, 1906 & Chachalaca colombiana & $\mathrm{E}$ & S.C-O.C-J.Z & $\begin{array}{c}2-5,13,15-17 \\
22,23\end{array}$ & C, D \\
\hline \multicolumn{6}{|l|}{ Odontophoridae } \\
\hline Colinus cristatus (Linnaeus, 1766) & Colín gorgiblanco & & S.C-O.C-J.Z & $4-6,18,22,23$ & C, D \\
\hline \multicolumn{6}{|l|}{ Podicipedidae } \\
\hline Podilymbus podiceps (Linnaeus, 1758) & Zampullín picogrueso & & O.C & & \\
\hline \multicolumn{6}{|l|}{ Columbidae } \\
\hline Columba livia Gmelin, 1789 & Paloma bravía & Int & S.C-O.C & 4,5 & \\
\hline Patagioenas cayennensis Bonnaterre, 1792 & Paloma colorada & & O.C & & $\mathrm{C}$ \\
\hline Patagioenas subvinacea (Lawrence, 1868) & Paloma vinosa & Alt, Loc, VU & O.C & & $\mathrm{C}$ \\
\hline Geotrygon montana (Linnaeus, 1758) & Paloma perdiz común & & O.C & & \\
\hline Leptotila verreauxi Bonaparte, 1855 & $\begin{array}{l}\text { Paloma montaraz } \\
\text { común }\end{array}$ & & S.C-O.C-J.Z & $\begin{array}{c}2,4-6,13,15- \\
17,22,23\end{array}$ & A, D \\
\hline Zenaida auriculata (des Murs, 1847) & Zenaida torcaza & & S.C-O.C-J.Z & $4,5,15-17,23$ & $\mathrm{D}$ \\
\hline Columbina passerina (Linnaeus, 1758) & Columbina común & & S.C-O.C-J.Z & $\begin{array}{l}5,6,15-17 \\
22,23\end{array}$ & A \\
\hline Columbina minuta (Linnaeus, 1766) & Columbina menuda & & S.C-O.C-J.Z & $\begin{array}{l}4-6,15-17 \\
22,23\end{array}$ & $\mathrm{C}$ \\
\hline Columbina talpacoti (Temminck, 1809) & Columbina colorada & & S.C-O.C-J.Z & $\begin{array}{c}2-6,13,15-17 \\
22,23\end{array}$ & C, D \\
\hline Claravis pretiosa (Ferrari-Pérez, 1886) & Tortolita azulada & & O.C & & \\
\hline \multicolumn{6}{|l|}{ Cuculidae } \\
\hline Crotophaga major Gmelin, 1788 & Garrapatero mayor & & O.C-J.Z & & A \\
\hline Crotophaga ani Linnaeus, 1758 & Garrapatero aní & & S.C-O.C-J.Z & $\begin{array}{c}2,4-6,13,15- \\
17,22,23\end{array}$ & $\mathrm{D}$ \\
\hline
\end{tabular}




\begin{tabular}{|c|c|c|c|c|c|}
\hline Crotophaga sulcirostris (Swainson, 1823) & Garrapatero asurcado & & O.C-J.Z & 17,22 & \\
\hline Tapera naevia (Linnaeus, 1766) & Cuclillo crespín & & S.C-O.C-J.Z & $\begin{array}{l}4,5,15-17 \\
22,23\end{array}$ & C, D \\
\hline Dromococcyx phasianellus Pelzeln, 1870 & Cuclillo faisán & & O.C & & \\
\hline Coсcyсиа minuta (Vieillot, 1817) & Cuco-ardilla menor & & O.C-J.Z & $17-19$ & \\
\hline Coccycua pumila Strickland, 1852 & Cuclillo enano & & O.C & & \\
\hline Piaya cayana (Linnaeus, 1766) & Cuco-ardilla común & & S.C-O.C-J.Z & $\begin{array}{l}2,4,5,13,15- \\
17,22,23\end{array}$ & $\mathrm{D}$ \\
\hline Coccyzus melacoryphus Vieillot, 1817 & Cuclillo canela & & S.C & 6 & \\
\hline Coccyzus americanus (Linnaeus, 1758) & Cuclillo piquigualdo & Lat, Trans, Irp, Mb & S.C-O.C-J.Z & $4,6,2,17,22$ & \\
\hline \multicolumn{6}{|l|}{ Nyctibiidae } \\
\hline Nyctibius griseus (Gmelin, 1789) & Biemparado común & & S.C & & \\
\hline \multicolumn{6}{|l|}{ Caprimulgidae } \\
\hline Chordeiles minor (Forster, 1771) & Añapero yanqui & Lat, Trans, Irp, Mb & O.C & & \\
\hline Chordeiles acutipennis (Hermann, 1783) & Chotacabras menor & Lat, Trans, Mb & & 19 & \\
\hline Systellura longirostris (Bonaparte, 1819) & Chotacabras serrana & & O.C & $4,17,18$ & $\mathrm{C}$ \\
\hline Nyctidromus albicollis (Gmelin, 1789) & Chotacabras pauraque & & S.C-J.Z & $4-6,15-17$ & $\mathrm{C}$ \\
\hline Hydropsalis cayennensis (Gmelin, 1789) & Chotacabras coliblanco & & S.C-J.Z & 18 & \\
\hline \multicolumn{6}{|l|}{ Apodidae } \\
\hline Streptoprocne rutila (Vieillot, 1817) & Vencejo cuellirrojo & & S.C-J.Z & $4,5,18,19,20$ & \\
\hline Streptoprocne zonaris (Shaw, 1796) & Vencejo acollarado & & S.C-O.C-J.Z & $\begin{array}{l}2,4-6,13,15- \\
17,23\end{array}$ & C, D \\
\hline Chaetura brachyura (Jardine, 1846) & Vencejo rabón & & O.C & 18,19 & \\
\hline $\begin{array}{l}\text { Aeronautes montivagus (d'Orbigny \& } \\
\text { Lafresnaye, 1837) }\end{array}$ & Vencejo montañés & & S.C-O.C-J.Z & $5,17,22$ & C, D \\
\hline \multicolumn{6}{|l|}{ Trochilidae } \\
\hline Florisuga mellivora (Linnaeus, 1758) & Colibrí nuquiblanco & Alt, Loc & S.C & 4 & \\
\hline Glaucis hirsutus (Gmelin, 1788) & Ermitaño hirsuto & & O.C & & \\
\hline Phaethornis anthophilus Bourcier, 1843 & Ermitaño ventripálido & & S.C-O.C & 4 & $\mathrm{C}$ \\
\hline Phaethornis guy (Lesson, 1832) & Ermitaño verde & Alt, Loc & $\mathrm{J} . \mathrm{Z}$ & & $\mathrm{C}$ \\
\hline Colibri delphinae (Lesson, 1839) & Colibrí pardo & Alt, Loc & O.C-J.Z & & $\mathrm{C}$ \\
\hline Colibri coruscans (Gould, 1846) & Colibrí rutilante & & O.C & 17 & \\
\hline $\begin{array}{l}\text { Colibri thalassinus cyanotus (Swainson, } \\
\text { 1823) }\end{array}$ & Colibrí verdemar & Alt, Loc & O.C & & B \\
\hline Heliothryx barroti (Bourcier 1843) & Colibrí hada occidental & & O.C & & \\
\hline Polytmus guainumbi Pallas, 1764 & Colibrí guainumbí & & O.C & & \\
\hline Chrysolampis mosquitus (Linnaeus) 1758 & Colibrí rubí & & S.C-O.C & 17 & \\
\hline
\end{tabular}




\begin{tabular}{|c|c|c|c|c|c|}
\hline Anthracothorax nigricollis (Vieillot) 1817 & Mango gorginegro & & S.C-O.C-J.Z & $4-6,15-17,23$ & A \\
\hline Adelomyia melanogenys (Fraser, 1840) & Colibrí jaspeado & & $\mathrm{J} . \mathrm{Z}$ & 19 & $\mathrm{~B}, \mathrm{C}$ \\
\hline Ocreatus underwoodii (Lesson, 1832) & Colibrí de raquetas & & O.C & & B \\
\hline $\begin{array}{l}\text { Heliomaster longirostris (Audebert and } \\
\text { Vieillot, 1801) }\end{array}$ & Colibrí piquilargo & Alt, Loc & O.C & & \\
\hline Chaetocercus mulsant (Bourcier, 1842) & Colibrí de Mulsant & & O.C & 17 & \\
\hline Chaetocercus heliodor (Bourcier, 1840) & Colibrí de Heliodoro & $\mathrm{CE}$ & S.C-O.C & & \\
\hline Chlorostilbon gibsoni (Fraser 1840) & Esmeralda de pico rojo & $\mathrm{CE}$ & S.C-O.C-J.Z & $4-6,17-19,23$ & A, C \\
\hline Chlorostilbon mellisugus Linnaeus, 1758 & Esmeralda de cola azul & & S.C-J.Z & $2,16,17$ & \\
\hline Chlorostilbon poortmani Bourcier, 1843 & Esmeralda colicorta & $\mathrm{CE}$ & S.C-O.C-J.Z & $\begin{array}{c}2,4-6,16-19 \\
22,23\end{array}$ & \\
\hline Campylopterus falcatus (Swainson, 1821) & Colibrí lazulita & $\mathrm{CE}$ & O.C & 17,18 & $\mathrm{~B}$ \\
\hline Thalurania colombica (Bourcier, 1843) & Zafiro coroniverde & Alt, Loc & O.C & & \\
\hline Amazilia tzacatl (De la Llave, 1833) & Amazilia colirrufa & & S.C-O.C-J.Z & $\begin{array}{c}2,4,5,6,15- \\
17,22,23\end{array}$ & \\
\hline Amazilia castaneiventris (Gould, 1856) & Amazilia ventricastaña & $\mathrm{E}, \mathrm{EN}(\mathrm{VU})$ & S.C-O.C-J.Z & $\begin{array}{l}1,2,4-11,15- \\
17,22,23\end{array}$ & C, D \\
\hline $\begin{array}{l}\text { Amazilia saucerrottei (DeLattre and } \\
\text { Bourcier, 1846) }\end{array}$ & Amazilia verdiazul & CE & O.C & 17 & \\
\hline Amazilia cyanifrons (Bourcier, 1843) & $\begin{array}{l}\text { Diamante de frente } \\
\text { azul }\end{array}$ & $\mathrm{E}$ & S.C-O.C-J.Z & $4-6,15-17$ & \\
\hline \multicolumn{6}{|l|}{ Aramidae } \\
\hline Aramus guarauna (Linnaeus, 1766) & Carrao & & O.C & & \\
\hline \multicolumn{6}{|l|}{ Rallidae } \\
\hline Aramides cajaneus Statius Muller, 1776 & Cotara chiricote & & O.C & & \\
\hline Anurolimnas viridis (Müller, 1776) & Polluela coronirrufa & & O.C & & \\
\hline Porzana carolina (Linnaeus, 1758) & Polluela sora & Lat, Trans, Irp, Mb & S.C-O.C & & \\
\hline Gallinula galeata (Lichtenstein, 1818) & Tagüita del norte & & S.C-O.C & 4 & \\
\hline Porphyrio martinica (Linnaeus, 1766) & $\begin{array}{l}\text { Calamoncillo } \\
\text { americano }\end{array}$ & Alt, Loc & S.C-O.C & 4 & A \\
\hline \multicolumn{6}{|l|}{ Charadriidae } \\
\hline Vanellus chilensis (Molina, 1782) & Avefría tero & & S.C-O.C-J.Z & $4,5,22$ & \\
\hline Charadrius vociferus Linnaeus, 1758 & Chorlitejo culirrojo & Lat, Trans, Irp, Mb & O.C & & \\
\hline \multicolumn{6}{|l|}{ Recurvirostridae } \\
\hline $\begin{array}{l}\text { Himantopus mexicanus (Statius Muller, } \\
\text { 1776) }\end{array}$ & $\begin{array}{l}\text { Cigüeñuela de cuello } \\
\text { negro }\end{array}$ & Lat, Trans, Mb & O.C & & \\
\hline \multicolumn{6}{|l|}{ Burhinidae } \\
\hline Burhinus bistriatus (Wagler, 1829) & Alcaraván venezolano & & O.C & & \\
\hline
\end{tabular}




\begin{tabular}{|c|c|c|c|c|c|}
\hline \multicolumn{6}{|l|}{ Scolopacidae } \\
\hline Calidris melanotos (Vieillot, 1819) & Correlimos pectoral & Lat, Trans, Mb & O.C & & \\
\hline Gallinago delicata (Ord, 1819) & Becasina & Lat, Trans, Mb & O.C & & \\
\hline Actitis macularius Linnaeus, 1766 & Andarríos maculado & Lat, Trans, Mb & O.C & & \\
\hline Tringa solitaria Wilson, 1813 & Andarríos solitario & Lat, Trans, Mb & S.C-O.C-J.Z & 4,22 & \\
\hline Tringa flavipes (Gmelin, 1789) & Patiamarillo chico & Lat, Trans, Mb & S.C & & \\
\hline \multicolumn{6}{|l|}{ Jacanidae } \\
\hline Jacana jacana (Linnaeus, 1766) & Jacana suramericana & & S.C-O.C & 4 & \\
\hline \multicolumn{6}{|l|}{ Laridae } \\
\hline Sternula superciliaris (Vieillot, 1819) & Charrancito amazónico & & O.C & & \\
\hline \multicolumn{6}{|l|}{ Phalacrocoracidae } \\
\hline Phalacrocorax brasilianus (Gmelin, 1789) & Cormorán biguá & Alt, Loc & O.C & & \\
\hline \multicolumn{6}{|l|}{ Anhingidae } \\
\hline Anhinga anhinga (Linnaeus, 1766) & Anhinga americana & & O.C & & \\
\hline \multicolumn{6}{|l|}{ Ardeidae } \\
\hline Tigrisoma lineatum (Boddaert, 1783) & Avetigre colorada & & O.C & & \\
\hline Cochlearius cochlearius (Linnaeus, 1766) & Martinete cucharón & & O.C & & \\
\hline Nycticorax nycticorax (Linnaeus, 1758) & Martinete común & & O.C-J.Z & 4 & \\
\hline Nyctanassa violacea (Linnaeus) 1758 & Martinete coronado & & O.C & & \\
\hline Butorides striata (Linnaeus, 1758) & Garcita azulada & & S.C-O.C-J.Z & 4,22 & \\
\hline Bubulcus ibis (Linnaeus, 1758) & Garcilla bueyera & Alt, Loc & S.C-O.C-J.Z & 4,5 & \\
\hline Ardea cocoi Linnaeus, 1766 & Garza cuca & & O.C & & \\
\hline Ardea alba Linnaeus, 1758 & Garceta grande & & S.C-O.C-J.Z & $2,13,22$ & \\
\hline Pilherodius pileatus (Boddaert, 1783) & Garza capirotada & & O.C & & \\
\hline Egretta thula (Molina, 1782) & Garceta nívea & Alt, Loc & O.C & & \\
\hline Egretta caerulea (Linnaeus, 1758) & Garceta azul & $\begin{array}{l}\text { Lat, Trans, Loc, } \\
\text { Irp, Mb }\end{array}$ & S.C-O.C-J.Z & 4 & \\
\hline \multicolumn{6}{|l|}{ Threskiornithidae } \\
\hline Phimosus infuscatus (Lichtenstein, 1823) & Ibis afeitado & & S.C-O.C-J.Z & 4 & \\
\hline \multicolumn{6}{|l|}{ Cathartidae } \\
\hline Cathartes aura (Linnaeus, 1758) & Aura gallipavo & & S.C-O.C-J.Z & $\begin{array}{c}2,4,5,13,15- \\
17,22,23\end{array}$ & $\mathrm{D}$ \\
\hline Cathartes burrovianus Cassin, 1845 & Aura sabanera & & O.C & 19 & $\mathrm{D}$ \\
\hline Coragyps atratus (Bechstein, 1783) & Zopilote negro & & S.C-O.C-J.Z & $\begin{array}{c}2,4,5,13,15- \\
17,22,23\end{array}$ & $\mathrm{D}$ \\
\hline
\end{tabular}




\begin{tabular}{|c|c|c|c|c|c|}
\hline \multicolumn{6}{|l|}{ Pandionidae } \\
\hline Pandion haliaetus (Linnaeus, 1758) & Águila pescadora & Lat, Trans, Mb & S.C-O.C & 4 & \\
\hline \multicolumn{6}{|l|}{ Accipitridae } \\
\hline Elanus leucurus (Vieillot, 1818) & Elanio maromero & & S.C-O.C & 4,23 & \\
\hline Gampsonyx swainsonii Vigors, 1819 & Elanio enano & & S.C-O.C-J.Z & $\begin{array}{l}2,5,13,15- \\
\quad 17,23\end{array}$ & A, D \\
\hline $\begin{array}{l}\text { Chondrohierax uncinatus (Temminck, } \\
\text { 1819) }\end{array}$ & Milano picogarfio & & S.C-O.C-J.Z & 17 & \\
\hline Busarellus nigricollis (Latham, 1790) & Busardo colorado & & O.C & & \\
\hline Rostrhamus sociabilis (Vieillot, 1817) & Caracolero común & & O.C & & \\
\hline Harpagus bidentatus (Latham, 1790) & Milano bidentado & & O.C & & \\
\hline Ictinia plumbea (Gmelin, 1788) & Elanio plomizo & & O.C & & \\
\hline Accipiter bicolor (Vieillot, 1817) & Azor bicolor & & O.C & 2 & \\
\hline Geranospiza caerulescens (Vieillot, 1817) & Azor zancón & & O.C & & \\
\hline Buteogallus urubitinga (Gmelin, 1788) & $\begin{array}{l}\text { Busardo-negro } \\
\text { urubitinga }\end{array}$ & & O.C & & \\
\hline Rupornis magnirostris (Gmelin, 1788) & Busardo caminero & & S.C-O.C-J.Z & $\begin{array}{c}2,4,5,13,15- \\
17,22,23\end{array}$ & C, D \\
\hline Parabuteo unicinctus (Temminck, 1824) & Busardo mixto & & S.C & & \\
\hline Geranoaetus albicaudatus (Vieillot, 1816) & Busardo coliblanco & & S.C-O.C-J.Z & 4,17 & $\mathrm{C}$ \\
\hline Geranoaetus melanoleucus (Vieillot, 1819) & Águila mora & & S.C-O.C-J.Z & 17,22 & \\
\hline Buteo nitidus (Latham, 1790) & Aguilucho gris & & O.C & 2,13 & \\
\hline Buteo platypterus (Vieillot, 1823) & Busardo aliancho & Lat, Trans, Mb & O.C & 4 & \\
\hline \multicolumn{6}{|l|}{ Tytonidae } \\
\hline Tyto alba (Scopoli, 1769) & Lechuza común & & O.C & & \\
\hline \multicolumn{6}{|l|}{ Strigidae } \\
\hline Megascops choliba (Vieillot, 1817) & Autillo chóliba & & S.C-O.C-J.Z & $4,6,22$ & A, C, D \\
\hline Asio stygius (Wagler, 1832) & Búho negruzco & & S.C-O.C & & \\
\hline \multicolumn{6}{|l|}{ Alcedinidae } \\
\hline Megaceryle torquata (Linnaeus, 1766) & $\begin{array}{l}\text { Martín gigante } \\
\text { neotropical }\end{array}$ & & S.C-O.C-J.Z & $2,13,22$ & $\mathrm{C}$ \\
\hline Chloroceryle amazona (Latham, 1790) & $\begin{array}{l}\text { Martín pescador } \\
\text { matraquero }\end{array}$ & & S.C & 4,6 & \\
\hline Chloroceryle americana (Gmelin, 1788) & Martín pescador verde & & S.C-O.C-J.Z & $4,6,18,22$ & \\
\hline Momotidae & & & & & A \\
\hline Momotus subrufescens P.L. Sclater, 1853 & Barrranquero ferina & & S.C-O.C-J.Z & $\begin{array}{c}2,4-6,13,15- \\
17,22,23\end{array}$ & C, D \\
\hline
\end{tabular}




\begin{tabular}{|c|c|c|c|c|c|}
\hline \multicolumn{6}{|l|}{ Galbulidae } \\
\hline Galbula ruficauda Cuvier, 1816 & Jacamará colirrufo & & O.C & & \\
\hline \multicolumn{6}{|l|}{ Picidae } \\
\hline Picumnus squamulatus Lafresnaye, 1854 & Carpinterito escamoso & & S.C-O.C-J.Z & 17 & \\
\hline Picumnus olivaceus (Lafresnaye, 1845) & Carpinterito oliváceo & & S.C-O.C-J.Z & $4,6,18,19,22$ & $\mathrm{C}$ \\
\hline Melanerpes rubricapillus (Cabanis, 1862) & Carpintero coronirrojo & & S.C-O.C-J.Z & $\begin{array}{c}2,4-6,15-17 \\
22,23\end{array}$ & A, C, D \\
\hline Veniliornis kirkii (Malherbe, 1845) & Carpintero culirrojo & & S.C-O.C-J.Z & 17 & $\mathrm{C}$ \\
\hline Colaptes rubiginosus (Swainson, 1818) & Carpintero oliváceo & & O.C & & \\
\hline Colaptes punctigula (Boddaert, 1783) & Carpintero moteado & & S.C-O.C-J.Z & $\begin{array}{l}2,13,17,19 \\
22\end{array}$ & $\mathrm{D}$ \\
\hline Dryocopus lineatus (Linnaeus, 1766) & Picamaderos listado & & O.C-J.Z & $4,5,18,22$ & $\mathrm{C}$ \\
\hline \multicolumn{6}{|l|}{ Falconidae } \\
\hline $\begin{array}{l}\text { Herpetotheres cachinnans (Linnaeus, } \\
1758 \text { ) }\end{array}$ & Halcón reidor & & O.C & & \\
\hline Caracara cheriway (Vieillot, 1817) & Carancho norteño & & O.C-J.Z & & \\
\hline Milvago chimachima (Vieillot, 1816) & Caracara chimachima & & S.C-O.C-J.Z & $\begin{array}{l}2,4,5,13 \\
15-17\end{array}$ & $\mathrm{C}$ \\
\hline Falco sparverius Linnaeus, 1758 & Cernícalo americano & & S.C-O.C-J.Z & $\begin{array}{l}2,4,5,13,15- \\
17,22\end{array}$ & $\mathrm{D}$ \\
\hline Falco columbarius Linnaeus, 1758 & Esmerejón & Lat, Trans, $\mathrm{Mb}$ & O.C & & \\
\hline Falco rufigularis Daudin, 1800 & Halcón murcielaguero & & $\mathrm{J} . \mathrm{Z}$ & 17 & \\
\hline Falco femoralis Temminck, 1819 & Halcón aleto & & S.C-J.Z & 18,22 & \\
\hline Falco peregrinus Tunstall, 1771 & Halcón peregrino & Lat, Trans, Mb & O.C & & \\
\hline \multicolumn{6}{|l|}{ Psittacidae } \\
\hline Brotogeris jugularis (Müller, 1776) & Catita churica & & O.C & & \\
\hline Pionus menstruus (Linnaeus, 1766) & Loro cabeciazul & & S.C-O.C & 5 & \\
\hline Amazona ochrocephala (Gmelin, 1788) & Amazona real & & S.C-J.Z & $4,5,18,22$ & \\
\hline Forpus conspicillatus (Lafresnaye, 1848) & Cotorrita de anteojos & $\mathrm{CE}$ & S.C-O.C-J.Z & $\begin{array}{l}2,4-6,13,15- \\
17,22,23\end{array}$ & C, D \\
\hline \multicolumn{6}{|l|}{ Thamnophilidae } \\
\hline $\begin{array}{l}\text { Thamnophilus multistriatus Lafresnaye, } \\
1844\end{array}$ & Batará crestibarrado & $\mathrm{CE}$ & S.C-O.C-J.Z & $\begin{array}{l}4-6,16-19 \\
22,23\end{array}$ & A, C, D \\
\hline $\begin{array}{l}\text { Thamnophilus atrinucha Salvin \& } \\
\text { Godman, } 1892\end{array}$ & $\begin{array}{l}\text { Batará pizarroso } \\
\text { occidental }\end{array}$ & & O.C & & \\
\hline Formicivora grisea (Boddaert, 1783) & Hormiguerito coicorita & & S.C-O.C-J.Z & $\begin{array}{c}2,4-6,13,17 \\
22,23\end{array}$ & $\mathrm{D}$ \\
\hline
\end{tabular}




\begin{tabular}{|c|c|c|c|c|c|}
\hline \multicolumn{6}{|l|}{ Furnariidae } \\
\hline Dendrocincla fuliginosa (Vieillot, 1818) & $\begin{array}{l}\text { Trepatroncos } \\
\text { fuliginoso }\end{array}$ & & O.C & & \\
\hline Dendroplex picus (Gmelin, JF, 1788) & $\begin{array}{l}\text { Trepatroncos } \\
\text { piquirrecto }\end{array}$ & & S.C-O.C-J.Z & $\begin{array}{l}2,4-6,13,15- \\
17,22,23\end{array}$ & $\mathrm{C}$ \\
\hline $\begin{array}{l}\text { Campylorhampus trochilirostris } \\
\text { (Lichtenstein, 1818) }\end{array}$ & Picoguadaña piquirrojo & & S.C & 6,17 & \\
\hline Xenops minutus (Sparrman, 1788) & Picolezna menudo & & O.C & & \\
\hline Synallaxis albescens Temminck, 1823 & Pijuí pechiblanco & & S.C-O.C-J.Z & $4-6,15-17$ & C, D \\
\hline \multicolumn{6}{|l|}{ Tyrannidae } \\
\hline $\begin{array}{l}\text { Phyllomyias griseiceps (Sclater \& Salvin, } \\
\text { 1871) }\end{array}$ & Mosquerito cabecigrís & & O.C & & \\
\hline Myiopagis gaimardii (d'Orbigny, 1840) & Fiofío selvático & & S.C-J.Z & 6,19 & \\
\hline Elaenia flavogaster (Thunberg, 1819) & Fiofío montano & & S.C-O.C-J.Z & $\begin{array}{l}4-6,15-17 \\
22,23\end{array}$ & $\mathrm{~B}, \mathrm{D}$ \\
\hline Elaenia parvirostris von Pelzeln, 1868 & Fiofío piquicorto & Lat, Trans, Ma & O.C & & \\
\hline Elaenia chiriquensis Lawrence, 1865 & Fiofío belicoso & Alt, Loc & S.C & $5,6,17,22$ & A, D \\
\hline Elaenia frantzii Lawrence, 1865 & Fiofío montano & Alt, Loc & S.C-J.Z & $4,6,17,18,22$ & $\mathrm{C}$ \\
\hline $\begin{array}{l}\text { Camptostoma obsoletum (Temminck, } \\
\text { 1824) }\end{array}$ & Mosquerito silbón & & S.C-O.C-J.Z & $\begin{array}{l}4-6,18,19 \\
22,23\end{array}$ & A \\
\hline Serpophaga cinerea (Tschudi, 1844) & Piojito guardarríos & & S.C-O.C & 4,17 & \\
\hline Phaeomyias murina (von Spix, 1819) & Piojito pardo & & S.C-O.C & & \\
\hline $\begin{array}{l}\text { Capsiempis flaveola (Lichtenstein, MHC, } \\
\text { 1823) }\end{array}$ & Mosquerito amarillo & & S.C-O.C & & \\
\hline Euscarthmus meloryphus zu Wied, 1831 & Tiranuelo copetón & & S.C-O.C-J.Z & $\begin{array}{l}4-6,15-17 \\
22,23\end{array}$ & C, D \\
\hline Mionectes olivaceus Lawrence, 1868 & Mosquero oliváceo & Alt, Loc & S.C & $2,4-6,18$ & $\mathrm{~B}, \mathrm{D}$ \\
\hline Mionectes oleagineus (Lichtenstein, 1823) & Mosquero aceitunado & Alt, Loc & O.C & 22 & \\
\hline Zimmerius chrysops (P.L. Sclater, 1859) & Tiranuelo cejiamarillo & & S.C-O.C & $4,17,19$ & $\mathrm{C}$ \\
\hline Leptopogon superciliaris (Tschudi, 1844) & Orejero coronigrís & & S.C-O.C-J.Z & $5,6,17$ & \\
\hline Lophotriccus pileatus (Tschudi, 1844) & Cimerillo andino & & O.C & & \\
\hline Atalotriccus pilaris (Cabanis, 1847) & Mosquerito ojiblanco & & O.C & 19 & \\
\hline $\begin{array}{l}\text { Hemitriccus margaritaceiventer } \\
\text { (d'Orbigny \& Lafresnaye, 1837) }\end{array}$ & Titirijí perlado & & S.C-O.C-J.Z & $\begin{array}{l}5,6,15-17 \\
22,23\end{array}$ & A, D \\
\hline Todirostrum cinereum (Linnaeus, 1766) & Titirijí común & & S.C-O.C-J.Z & $\begin{array}{c}2,4-6,13,15- \\
17,22,23\end{array}$ & $\mathrm{D}$ \\
\hline $\begin{array}{l}\text { Rhynchocyclus olivaceus (Temminck, } \\
\text { 1818) }\end{array}$ & Picoplano oliváceo & & S.C & & \\
\hline $\begin{array}{l}\text { Tolmomyias sulphurescens (von Spix, } \\
\text { 1821) }\end{array}$ & Picoplano sulfuroso & & S.C-O.C-J.Z & $15-17,22,23$ & A, C, D \\
\hline
\end{tabular}




\begin{tabular}{|c|c|c|c|c|c|}
\hline Myiophobus flavicans (P.L. Sclater, 1860) & Mosquero amarillo & & S.C & 17 & \\
\hline Myiophobus fasciatus (Müller, 1776) & Mosquero estriado & & S.C & $5,6,17,18,23$ & \\
\hline Hirundinea ferruginea (Gmelin, JF, 1788) & $\begin{array}{l}\text { Atrapamoscas de } \\
\text { precipicios }\end{array}$ & & S.C-J.Z & & \\
\hline Nephelomyias pulcher (P.L. Sclater, 1861) & Mosquero hermoso & & O.C & & \\
\hline Cnemotriccus fuscatus (zu Wied, 1831) & Mosquero parduzco & & O.C & & \\
\hline Empidonax traillii (Audubon, 1828) & Mosquero saucero & Lat, Trans, Mb & S.C-O.C & & \\
\hline Empidonax alnorum Brewster, 1895 & Mosquero alisero & Lat, Trans, Mb & S.C-O.C & 6 & \\
\hline Contopus cooperi (Swainson, 1832) & Pibí boreal & Lat, Trans, Mb, NT & S.C-O.C-J.Z & 5 & \\
\hline $\begin{array}{l}\text { Contopus fumigatus (d'Orbigny \& } \\
\text { Lafresnaye, 1837) }\end{array}$ & Pibí ahumado & & S.C-O.C & 17,22 & \\
\hline Contopus sordidulus P.L. Sclater, 1859 & Pibí occidental & Lat, Trans, Mb & S.C-O.C & & \\
\hline Contopus virens (Linnaeus, 1766) & Pibí oriental & Lat, Trans, Mb & S.C-O.C & 4 & \\
\hline Contopus cinereus (Spix, 1819) & Pibí tropical & & O.C-J.Z & 17 & $\mathrm{C}$ \\
\hline Sayornis nigricans (Swainson, 1823) & Mosquero negro & & S.C-O.C-J.Z & $\begin{array}{c}2,4,6,13,18 \\
19,22\end{array}$ & A \\
\hline Pyrocephalus rubinus (Boddaert, 1783) & Mosquero cardenal & & S.C-O.C-J.Z & $15-17,22,23$ & A, D \\
\hline Myiozetetes cayanensis (Linnaeus, 1766) & Bienteveo alicastaño & & S.C-O.C-J.Z & $\begin{array}{l}2,4-6,13,15- \\
17,22,23\end{array}$ & $\mathrm{~A}$ \\
\hline Myiozetetes similis (Spix, 1819) & Bienteveo sociable & Alt, Loc & O.C & 22 & $\mathrm{D}$ \\
\hline Pitangus sulphuratus (Linnaeus, 1766) & Bienteveo común & & S.C-O.C-J.Z & $\begin{array}{l}4-6,15-17 \\
22,23\end{array}$ & C, D \\
\hline Legatus leucophaius (Vieillot, 1818) & Atrapamoscas pirata & & & 17 & B \\
\hline Machetornis rixosa (Vieillot, 1819) & Picabuey & & S.C-O.C-J.Z & $\begin{array}{l}4,5,15-17 \\
22,23\end{array}$ & A, D \\
\hline $\begin{array}{l}\text { Myiodynastes chrysocephalus } \\
\text { (von Tschudi, 1844) }\end{array}$ & $\begin{array}{l}\text { Bienteveo } \\
\text { coronodorado }\end{array}$ & & O.C & & \\
\hline Myiodynastes maculatus (Müller, 1776) & Bienteveo rayado & Lat, Trans, Ma & S.C-O.C-J.Z & $\begin{array}{c}2,5,13,15-17 \\
22,23\end{array}$ & C, D \\
\hline Megarynchus pitangua (Linnaeus, 1766) & Bienteveo pitanguá & & S.C-O.C-J.Z & $2,4,6,18,22$ & $\mathrm{C}$ \\
\hline Empidonomus varius (Vieillot, 1818) & Tuquito rayado & Lat, Trans, Ma & S.C & & \\
\hline Tyrannus melancholicus Vieillot, 1819 & Tirano melancólico & Alt, Loc & S.C-O.C-J.Z & $\begin{array}{l}2,4,5,13,15- \\
\quad 17,22,23\end{array}$ & A, C, D \\
\hline Tyrannus savana Vieillot, 1808 & Tijereta sabanera & Lat, Trans, Irp, Ma & S.C-O.C & 4 & \\
\hline $\begin{array}{l}\text { Myiarchus tuberculifer (d'Orbigny \& } \\
\text { Lafresnaye, 1837) }\end{array}$ & Copetón capirotado & & S.C-O.C-J.Z & 18,19 & \\
\hline Myiarchus apicalis Sclater \& Salvin, 1881 & Copetón apical & $\mathrm{E}$ & S.C-O.C-J.Z & $\begin{array}{l}2,4-6,13,15- \\
17,22,23\end{array}$ & A, C, D \\
\hline Myiarchus tyrannulus (Müller, 1776) & Copetón tiranillo & & S.C-J.Z & $4,15-17$ & $\mathrm{D}$ \\
\hline
\end{tabular}




\begin{tabular}{|c|c|c|c|c|c|}
\hline \multicolumn{6}{|l|}{ Pipridae } \\
\hline Machaeropterus regulus (Hahn, 1819) & Manaquin franjeado & & S.C & & \\
\hline \multicolumn{6}{|l|}{ Tityridae } \\
\hline Pachyramphus rufus (Boddaert, 1783) & Anambé cinéreo & & S.C-O.C & 17,22 & A \\
\hline $\begin{array}{l}\text { Pachyramphus polychopterus (Vieillot, } \\
\text { 1818) }\end{array}$ & Anambé aliblanco & & O.C & & \\
\hline \multicolumn{6}{|l|}{ Vireonidae } \\
\hline Cyclarhis gujanensis (Gmelin, 1789) & Vireón cejirrufo & & S.C-O.C-J.Z & $\begin{array}{l}4,6,13,15- \\
\quad 17,22\end{array}$ & A, C \\
\hline Hylophilus flavipes Lafresnaye, 1845 & Verdillo paticlaro & & S.C-O.C-J.Z & $6,15-17,22$ & C, D \\
\hline Vireo leucophrys (Lafresnaye, 1844) & Verderón montañero & & & $15-17$ & \\
\hline Vireo olivaceus (Lafresnaye, 1844) & Vireo chiví & $\begin{array}{l}\text { "Lat, Trans, Irp } \\
\text { Mb" }\end{array}$ & S.C-O.C-J.Z & $\begin{array}{l}2,4,6,13,15- \\
17,22\end{array}$ & \\
\hline Vireo flavoviridis (Cassin, 1851) & Vireo verdiamarillo & $\begin{array}{l}\text { "Lat, Trans, Irp } \\
\text { Mb" }\end{array}$ & O.C & & \\
\hline \multicolumn{6}{|l|}{ Hirundinidae } \\
\hline Pygochelidon cyanoleuca (Vieillot, 1817) & $\begin{array}{l}\text { Golondrina } \\
\text { barranquera }\end{array}$ & Lat, Trans, Irp & S.C-O.C-J.Z & $4,15-17$ & $\mathrm{~B}$ \\
\hline Orochelidon murina (Cassin, 1853) & $\begin{array}{l}\text { Golondrina } \\
\text { ventriparda }\end{array}$ & & O.C & 2,4 & \\
\hline Stelgidopteryx ruficollis (Vieillot, 1817) & Golondrina gorgirrufa & & S.C-O.C-J.Z & $\begin{array}{l}2,4-6,13,15- \\
17,23\end{array}$ & $\mathrm{D}$ \\
\hline Progne tapera (Linnaeus, 1766) & Golondrina parda & Lat, Trans, Ma & S.C-O.C & 4,17 & \\
\hline Tachycineta albiventer (Boddaert, 1783) & Golondrina aliblanca & & S.C & & \\
\hline Riparia riparia (Linnaeus, 1758) & Avión zapador & Lat, Trans, Mb & O.C & 4 & \\
\hline Hirundo rustica Linnaeus, 1758 & Golondrina común & Lat, Trans, Irp , Mb & S.C-O.C-J.Z & $2,13,17,18$ & \\
\hline \multicolumn{6}{|l|}{ Troglodytidae } \\
\hline Troglodytes aedon Vieillot 1809 & Chercán & & S.C-O.C-J.Z & $\begin{array}{l}2,4-6,13,15- \\
17,22,23\end{array}$ & C, D \\
\hline $\begin{array}{l}\text { Campylorhynchus griseus bicolor (von } \\
\text { Pelzeln, 1875) }\end{array}$ & $\begin{array}{l}\text { Cucarachero } \\
\text { currucuchú }\end{array}$ & $\mathrm{E}$ & S.C-O.C-J.Z & $\begin{array}{l}2,4-6,13,15- \\
17,22,23\end{array}$ & A, C, D \\
\hline $\begin{array}{l}\text { Thryophilus nicefori Meyer de } \\
\text { Schauensee, } 1946\end{array}$ & $\begin{array}{l}\text { Cucarachero de } \\
\text { nicéforo }\end{array}$ & $\mathrm{E}, \mathrm{CR}(\mathrm{EN}-\mathrm{VU})$ & S.C-O.C-J.Z & $\begin{array}{l}6,12-14,17- \\
19,22\end{array}$ & C \\
\hline \multicolumn{6}{|l|}{ Polioptilidae } \\
\hline Polioptila plumbea (Gmelin, 1788) & Perlita tropical & & O.C & & \\
\hline \multicolumn{6}{|l|}{ Turdidae } \\
\hline Catharus fuscescens (Stephens, 1817) & Zorzalito rojizo & Lat, Trans, Mb & S.C & 4 & \\
\hline Catharus minimus (Lafresnaye, 1848) & Zorzal carigrís & Lat, Trans, Mb & & 19 & C, D \\
\hline Catharus ustulatus (Nuttall, 1840) & Zorzalito de Swainson & Lat, Trans, Mb & S.C-O.C-J.Z & $\begin{array}{c}2,4,6,13,17 \\
18,22\end{array}$ & \\
\hline
\end{tabular}




\begin{tabular}{|c|c|c|c|c|c|}
\hline Turdus flavipes (Vieillot, 1818) & Mirlo azulado & Alt, Loc & S.C & $4,18,22$ & $\mathrm{C}$ \\
\hline Turdus leucomelas Vieillot 1818 & Zorzal sabiá & & S.C-J.Z & $\begin{array}{l}4-6,15-17 \\
22,23\end{array}$ & C, D \\
\hline Turdus ignobilis P.L. Sclater, 1857 & Zorzal piquinegro & & S.C-O.C-J.Z & $\begin{array}{c}2,4,5,13,15- \\
17,23\end{array}$ & $\mathrm{D}$ \\
\hline \multicolumn{6}{|l|}{ Mimidae } \\
\hline Mimus gilvus (Vieillot, 1808) & Sinsonte tropical & & S.C-O.C-J.Z & $\begin{array}{c}2,4,5,13,15- \\
17,22,23\end{array}$ & A, C, D \\
\hline \multicolumn{6}{|l|}{ Thraupidae } \\
\hline Chlorophanes spiza (Linnaeus, 1758) & Mielerito verde & & O.C & & \\
\hline Sicalis flaveola (Sparrman, 1789) & Chirigüe azafranado & & S.C-O.C-J.Z & $\begin{array}{c}4-6,13,15-17 \\
22,23\end{array}$ & $\mathrm{D}$ \\
\hline Volatinia jacarina (Linnaeus, 1766) & Semillero volatinero & & S.C-O.C-J.Z & $\begin{array}{c}2,13,15-17 \\
22\end{array}$ & C, D \\
\hline Eucometis penicillata (Spix, 1821) & Tángara cabecigrís & & O.C & & \\
\hline Tachyphonus rufus (Boddaert, 1783) & Tángara negra & & S.C-O.C & $5,17,19,23$ & \\
\hline Ramphocelus dimidiatus Lafresnaye, 1837 & Tángara dorsirroja & CE & S.C-O.C-J.Z & $\begin{array}{c}2,4-6,13,17 \\
18,22,23\end{array}$ & $\mathrm{C}$ \\
\hline Cyanerpes cyaneus (Linnaeus, 1766) & Mielerito patirrojo & & S.C & 4,22 & \\
\hline Sporophila minuta (Linnaeus, 1758) & Semillero pechirrufo & & S.C-O.C-J.Z & 17,22 & \\
\hline Sporophila angolensis (Linnaeus, 1766) & Semillero curió & & O.C & & \\
\hline Sporophila nigricollis (Vieillot, 1823) & $\begin{array}{c}\text { Semillero } \\
\text { ventriamarillo }\end{array}$ & & S.C-O.C-J.Z & $\begin{array}{l}2,4-6,13,17 \\
18,22,23\end{array}$ & \\
\hline Sporophila schistacea Lawrence, 1862 & Semillero pizarroso & & S.C-O.C & 6 & \\
\hline Saltator maximus (Statius Muller, 1776) & Pepitero gorgicanelo & & S.C-O.C-J.Z & 6,22 & \\
\hline Saltator coerulescens Vieillot 1817 & Pepitero grisáceo & & S.C-O.C-J.Z & $2,17,22$ & $\mathrm{C}$ \\
\hline Saltator striatipectus Lafresnaye, 1847 & Pepitero listado & & S.C-O.C-J.Z & $15-17,22$ & $\mathrm{~A}, \mathrm{C}$ \\
\hline Emberizoides herbicola (Vieillot, 1817) & Coludo colicuña & & O.C & & \\
\hline Coereba flaveola (Linnaeus, 1758) & Platanero & & O.C-J.Z & $\begin{array}{l}2,4-6,13,15- \\
17,22,23\end{array}$ & $\mathrm{C}$ \\
\hline Tiaris olivaceus (Linnaeus, 1766) & Semillero tomeguín & & S.C-O.C-J.Z & $\begin{array}{l}2,4-6,13,17 \\
18,22,23\end{array}$ & \\
\hline Tiaris bicolor (Linnaeus, 1766) & Semillero biocolor & & S.C-J.Z & $\begin{array}{l}2,4-6,13,15- \\
17,22,23\end{array}$ & A, C, D \\
\hline Schistochlamys melanopis (Latham, 1790) & Tángara carinegra & & S.C-J.Z & 4,22 & \\
\hline Stilpnia heinei (Cabanis, 1850) & Tángara coroninegra & & S.C-O.C & $4,17,22$ & \\
\hline Stilpnia vitriolina (Cabanis, 1850) & Tángara matorralera & CE & S.C-O.C-J.Z & $\begin{array}{c}2,4-6,13,15- \\
17,22,23\end{array}$ & A, D \\
\hline $\begin{array}{l}\text { Stilpnia cyanicollis (d'Orbigny \& } \\
\text { Lafresnaye, 1837) }\end{array}$ & Tángara cabeciazul & & S.C-O.C-J.Z & $4-6,17,18,22$ & \\
\hline Tángara gyrola (Linnaeus, 1758) & Tángara cabecibaya & & O.C-J.Z & & \\
\hline
\end{tabular}




\begin{tabular}{|c|c|c|c|c|c|}
\hline Thraupis episcopus (Linnaeus, 1766) & Tángara azuleja & & S.C-O.C-J.Z & $\begin{array}{l}2,4-6,13,15- \\
17,22,23\end{array}$ & A, D \\
\hline Thraupis palmarum (Wied, 1821) & Tángara palmera & & S.C-O.C-J.Z & $\begin{array}{l}2,4-6,13,15- \\
17,22,23\end{array}$ & A, D \\
\hline \multicolumn{6}{|l|}{ Emberizidae } \\
\hline Ammodramus humeralis (Bosc, 1792) & Chingolo pajonalero & & O.C & & \\
\hline Arremon atricapillus (Lawrence, 1874) & $\begin{array}{l}\text { Gorrión montés } \\
\text { cabecinegro }\end{array}$ & $\mathrm{CE}$ & O.C-J.Z & 22 & \\
\hline $\begin{array}{l}\text { Arremon schlegeli canidorsum J.T. } \\
\text { Zimmer, } 1941\end{array}$ & Cerquero alidorado & $\mathrm{E}, \mathrm{VU}$ & S.C-O.C-J.Z & $\begin{array}{l}2,5,6,13,15- \\
17,22\end{array}$ & A, D \\
\hline Zonotrichia capensis (Müller, 1776) & Chingolo común & & S.C-O.C & $2,4,17,18$ & B \\
\hline \multicolumn{6}{|l|}{ Cardinalidae } \\
\hline Piranga flava (Vieillot, 1819) & Piranga bermeja & Alt, Loc & S.C-J.Z & $2,13,15-17$ & $\mathrm{D}$ \\
\hline Piranga rubra (Linnaeus, 1758) & Piranga roja & Lat, Trans, Irp, Mb & S.C-O.C-J.Z & $\begin{array}{l}2,4,6,13,15- \\
17,22\end{array}$ & A \\
\hline Piranga olivacea (Gmelin, 1789) & Piranga escarlata & Lat, Trans, Mb & S.C-J.Z & $4,6,17,22$ & \\
\hline $\begin{array}{l}\text { Pheucticus aureoventris (Orbigny \& } \\
\text { Lafresnaye, 1837) }\end{array}$ & Picogrueso dorsinegro & & O.C & 22 & \\
\hline Pheuticus ludovicianus (Linnaeus, 1766) & $\begin{array}{l}\text { Picogrueso } \\
\text { pechirrosado }\end{array}$ & Lat, Trans, Mb & S.C-J.Z & 4,17 & A \\
\hline \multicolumn{6}{|l|}{ Parulidae } \\
\hline Parkesia noveboracensis (Gmelin, 1789) & $\begin{array}{c}\text { Reinita charquera } \\
\text { norteña }\end{array}$ & Lat, Trans, Mb & S.C-O.C-J.Z & $\begin{array}{c}2,4,6,13,14 \\
15-17\end{array}$ & \\
\hline Mniotilta varia (Linnaeus, 1766 ) & Reinita trepadora & Lat, Trans, Mb & S.C-O.C-J.Z & 19,22 & \\
\hline Protonotaria citrea (Boddaert, 1783) & Reinita protonotaria & Lat, Trans, Mb & S.C-O.C & 6,17 & \\
\hline Leiothlypis peregrina (Wilson, 1811) & Reinita de Tennessee & Lat, Trans, Mb & S.C-O.C-J.Z & $4,6,17$ & \\
\hline Oporornis agilis (Wilson, 1812) & Reinita de Connecticut & Lat, Trans, Mb & S.C & 4,6 & \\
\hline Geothlypis philadelphia (Wilson, 1810) & Reinita plañidera & Lat, Trans, Mb & S.C & $4,6,17$ & \\
\hline Setophaga ruticilla (Linnaeus, 1758) & Candelita norteña & Lat, Trans, Mb & S.C-O.C & & B \\
\hline Setophaga cerulea (Wilson, 1810) & Reinita cerúlea & Lat, Trans, Mb, VU & S.C & & $\mathrm{C}$ \\
\hline Setophaga americana (Linnaeus, 1758) & Parula norteña & Lat, Trans, Mb & O.C & & \\
\hline Setophaga pitiayumi (Vieillot, 1817) & Parula pitiayumí & Alt, Loc & S.C-O.C-J.Z & $\begin{array}{c}2,4-6,13,15- \\
17,22,23\end{array}$ & $\mathrm{C}$ \\
\hline Setophaga castanea (Wilson, 1810) & Reinita castaña & Lat, Trans, Mb & O.C & & $\mathrm{C}$ \\
\hline Setophaga fusca (Müller, 1776) & Reinita gorginaranja & Lat, Trans, Mb & S.C-O.C-J.Z & $4,6,17,19,22$ & $\mathrm{D}$ \\
\hline Setophaga petechia (Linnaeus, 1766) & Reinita de manglar & Lat, Trans, Irp, Mb & S.C-O.C-J.Z & $4,6,17,19$ & $\mathrm{D}$ \\
\hline Setophaga striata (Forster, 1772) & Reinita estriada & Lat, Trans, Mb & S.C-O.C-J.Z & $16-19,22$ & C, D \\
\hline Myiothlypis cinereicollis P.L. Sclater, 1864 & Reinita gorgigrís & CE, NT & O.C & & \\
\hline
\end{tabular}




\begin{tabular}{|c|c|c|c|c|c|}
\hline Basileuterus rufifrons (Swainson, 1838) & Reinita coronirrufa & & S.C-O.C-J.Z & $\begin{array}{c}2,5,6,13,15- \\
17,22,23\end{array}$ & $\mathrm{C}$ \\
\hline Basileuterus culicivorus (Deppe, 1830) & Reinita coronidorada & & S.C-J.Z & $17,18,22$ & $\mathrm{C}$ \\
\hline Cardellina canadensis (Linnaeus, 1766) & Reinita canadiense & Lat, Trans, Mb & S.C-O.C-J.Z & 4,6 & \\
\hline \multicolumn{6}{|l|}{ Icteridae } \\
\hline Icterus icterus (Hartert, E, 1902) & Turpial venezolano & VU & S.C-J.Z & $\begin{array}{l}5,6,17,19 \\
22,23\end{array}$ & $\mathrm{C}$ \\
\hline Icterus mesomelas (Wagler, 1829) & Turpial coliamarillo & & S.C-J.Z & 22 & \\
\hline Icterus auricapillus Cassin, 1848 & Turpial coroninaranja & & S.C-J.Z & 22 & \\
\hline Icterus chrysater (Lesson, 1844) & Turpial dorsidorado & & S.C-O.C-J.Z & $\begin{array}{l}2,4,13,15- \\
\quad 17,22\end{array}$ & A, C, D \\
\hline Icterus galbula (Linnaeus, 1758) & Turpial de Baltimore & Lat, Trans, Mb & S.C-J.Z & 6 & \\
\hline Icterus nigrogularis (Hahn, 1819) & Turpial amarillo & & S.C-O.C-J.Z & $\begin{array}{l}2,5,6,13,15- \\
17,22,23\end{array}$ & $\mathrm{D}$ \\
\hline Molothrus bonariensis (Gmelin, 1789) & Tordo renegrido & & S.C-O.C & $2,13,17,18$ & A \\
\hline Quiscalus lugubris Swainson, 1838 & Zanate caribeño & & S.C & 22 & \\
\hline Quiscalus mexicanus (Gmelin, 1788) & Zanate mexicano & & O.C & & \\
\hline Sturnella magna (Linnaeus, 1758) & Turpial oriental & & S.C-O.C-J.Z & $4,5,17$ & \\
\hline \multicolumn{6}{|l|}{ Fringillidae } \\
\hline Spinus spinescens (Bonaparte, 1850) & Jilguero andino & CE & O.C & & \\
\hline Spinus psaltria (Say, 1823) & Jilguero menor & & S.C-O.C & $4,17,18$ & \\
\hline $\begin{array}{l}\text { Euphonia laniirostris (Orbigny \& } \\
\text { Lafresnaye, 1837) }\end{array}$ & Eufonia piquigruesa & & S.C-O.C-J.Z & $\begin{array}{l}2,4,6,13,15- \\
17,22,23\end{array}$ & A \\
\hline Euphonia cyanocephala (Vieillot, 1818) & Eufonia culidorada & & S.C-O.C & & $\mathrm{C}$ \\
\hline
\end{tabular}

Numeración de referencia de fuentes bibliográficas y bases de datos consultadas para la elaboración del listado de las aves del Cañón del Chicamocha, Colombia.

\begin{tabular}{ll}
\hline Referencias & Número \\
\hline Chaves-Portilla \& Cortés-Herrera (2006) & 1 \\
\hline Ciri et al. (2011) & 2 \\
\hline Collar et al. (1992) & 3 \\
\hline Collazos-González (2015) & 4 \\
\hline Collazos-González (2016) & 5 \\
\hline Collazos-González \& Echeverry-Galvis (2017) & 6 \\
\hline Cortés-Herrera (2006) & 7 \\
\hline Cortés-Herrera et al. (2004) & 8 \\
\hline
\end{tabular}




\begin{tabular}{|c|c|}
\hline Cortés-Herrera et al. (2007) & 9 \\
\hline Cortés-Herrera \& Briceño-Buitrago (2007) & 10 \\
\hline Hernández-Jaramillo et al. (2007) & 11 \\
\hline López-Lanús \& Cadena (2002) & 12 \\
\hline Melo et al. (2012) & 13 \\
\hline Meyer de Schauensee (1946) & 14 \\
\hline Parra et al. (2005) & 15 \\
\hline Parra et al. (2006) & 16 \\
\hline Parra et al. (2010) & 17 \\
\hline Rico-Guevara (2018) & 18 \\
\hline Serrano et al. (2009) & 19 \\
\hline Valderrama (2005) & 20 \\
\hline Valderrama et al. (2007a) & 21 \\
\hline Valderrama et al. (2008) & 22 \\
\hline Valderrama et al. (2007b) & 23 \\
\hline Valderrama \& Páez (2009) & 24 \\
\hline Vargas-Moreno (2007) & 25 \\
\hline Zuluaga-Carrero (2017) & 26 \\
\hline Orozco-Romero (2018) & 27 \\
\hline Bases de datos & Letra \\
\hline $\mathrm{ICN}$ & A \\
\hline $\mathrm{IAvH}$ & B \\
\hline Xeno-canto & $\mathrm{C}$ \\
\hline GBIF - Montealegre (2018) & $\mathrm{D}$ \\
\hline
\end{tabular}

\section{Acrónimos usados}

Hábitos de migración: Migratorio boreal (Mb), Migratoria austral (Ma), Transfronteriza (Trans), Latitudinal (Lat), Altitudinal (Alt), Loc (Local), Invernante con Poblaciones (Irp); Introducida (Int). Las categorías de amenaza a nivel nacional siguen a Renjifo et al. (2014, 2016): En Peligro Crítico CR, En Peligro EN, Vulnerable VU, Casi Amenazado NT, Preocupación Menor LC. Las mismas categorías de amenaza se emplean a nivel global según IUCN (2016) y se establecen en paréntesis si difieren de la nacional. Obs. Autores: Sergio Collazos (SC), Juliana Zuluaga (JZ) y Oswaldo Cortes (OC). 
Sergio Andrés Collazos-González

Pontificia Universidad Javeriana

Bogotá, Colombia

collazoss@javeriana.edu.co

https://orcid.org/0000-0001-7822-2250

Juliana Zuluaga-Carrero

Jardín Botánico de Bogotá

Bogotá, Colombia

zuluaga.juliana@javeriana.edu.co

https://orcid.org/0000-0002-9208-5958

\section{José Oswaldo Cortés-Herrera}

Universidad Distrital Francisco José de Caldas

Bogotá, Colombia

oswaldo.aves@gmail.com

https://orcid.org/0000-0001-9205-4898
Aves del Cañón del Chicamocha, Colombia: un llamado para su conservación

Citación del artículo: Collazos-González, S.A., Zuluaga, J. \& Cortés, J.O. (2020). Aves del Cañón del Chicamocha, Colombia: un llamado para su conservación. Biota Colombiana, 21(1), 58-85. DOI: 10.21068/c2020.v21n01a05.

Recibido: 10 de junio 2019

Aprobado: 12 de noviembre 2019 